\title{
Uranus Pathfinder: exploring the origins and evolution of Ice Giant planets
}

Christopher S. Arridge • Craig B. Agnor • Nicolas André • Kevin H. Baines • Leigh N. Fletcher • Daniel Gautier • Mark D. Hofstadter • Geraint H. Jones • Laurent Lamy • Yves Langevin • Olivier Mousis • Nadine Nettelmann • Christopher T. Russell • Tom Stallard • Matthew S. Tiscareno • Gabriel Tobie - Andrew Bacon - Chris Chaloner • Michael Guest • Steve Kemble • Lisa Peacocke • Nicholas Achilleos - Thomas P. Andert • Don Banfield • Stas Barabash • Mathieu Barthelemy • Cesar Bertucci • Pontus Brandt • Baptiste Cecconi • Supriya Chakrabarti • Andy F. Cheng • Ulrich Christensen - Apostolos Christou • Andrew J. Coates • Glyn Collinson • John F. Cooper • Regis Courtin • Michele K. Dougherty • Robert W. Ebert • Marta Entradas • Andrew N. Fazakerley • Jonathan J. Fortney • Marina Galand • Jaques Gustin • Matthew Hedman • Ravit Helled • Pierre Henri • Sebastien Hess • Richard Holme •

Özgur Karatekin • Norbert Krupp · Jared Leisner • Javier Martin-Torres • Adam Masters • Henrik Melin • Steve Miller • Ingo Müller-Wodarg • Benoît Noyelles • Chris Paranicas • Imke de Pater • Martin Pätzold • Renée Prangé · Eric Quémerais • Elias Roussos • Abigail M. Rymer • Agustin Sánchez-Lavega - Joachim Saur • Kunio M. Sayanagi • Paul Schenk • Gerald Schubert • Nick Sergis • Frank Sohl • Edward C. Sittler Jr. • Nick A. Teanby • Silvia Tellmann • Elizabeth P. Turtle • Sandrine Vinatier • Jan-Erik Wahlund • Philippe Zarka

\footnotetext{
N. Achilleos

Department of Physics and Astronomy, University College London, London, UK

School of Physics and Astronomy, Queen Mary University of London, London, UK
}

T. P. Andert

Universität der Bundeswehr, Munich, Germany

N. André

Centre d'Etude Spatiale des Rayonnements / CNRS, Toulouse, France

C. S. Arridge · G. H. Jones · A. J. Coates · A. N. Fazakerley · A. Masters

Mullard Space Science Laboratory, Department of Space and Climate Physics, University

College London, London, UK 
Abstract The "Ice Giants" Uranus and Neptune are a different class of planet compared to Jupiter and Saturn. Studying these objects is important for furthering our understanding of the formation and evolution of the planets, and unravelling the fundamental physical and chemical processes in the Solar System. The importance of filling these gaps in our knowledge of the Solar System is particularly acute when trying to apply our understanding to the numerous planetary systems that have been discovered around other stars. The Uranus Pathfinder (UP) mission thus represents the quintessential aspects of the objectives of the European planetary community as expressed in ESA's Cosmic Vision 2015-2025. UP was proposed to the European Space Agency's M3 call for medium-class missions in 2010 and proposed to be the first orbiter of an Ice Giant planet. As the most accessible Ice Giant within the M-class mission envelope Uranus was identified as the mission target. Although not selected for this call the UP mission concept provides a baseline framework for the exploration of Uranus with existing low-cost platforms and underlines

C. S. Arridge $(\bowtie) \cdot$ G. H. Jones · A. J. Coates · A. N. Fazakerley · A. Masters

The Centre for Planetary Sciences at UCL/Birkbeck, London, UK

e-mail: csa@mssl.ucl.ac.uk

A. Bacon · C. Chaloner · M. Guest

Systems Engineering and Asssessment Ltd., Bristol, UK

K. H. Baines · M. D. Hofstadter

NASA Jet Propulsion Laboratory, Pasadena, CA, USA

M. S. Tiscareno $\cdot$ D. Banfield $\cdot$ M. Hedman

Cornell University, Ithaca, NY, USA

S. Barabash

Swedish Institute of Space Physics, Kiruna, Sweden

M. Barthelemy

Université Joseph Fourier/CNRS-INSU / Institut de Planétologie et d'Astrophysique de Grenoble (IPAG), Grenoble, France

C. Bertucci

Institute of Astronomy and Space Physics, University of Buenos Aires, Buenos Aires, Argentina

P. Brandt · A. F. Cheng · C. Paranicas · A. M. Rymer · E. P. Turtle

Johns Hopkins University Applied Physics Laboratory, Laurel, MD, USA

D. Gautier · L. Lamy · B. Cecconi · R. Courtin · P. Henri · R. Prangé · S. Vinatier · P. Zarka

LESIA, CNRS-Observatoire de Paris, Meudon, France

S. Chakrabarti

Centre for Space Physics, Boston University, Boston, MA, USA

U. Christensen · N. Krupp · E. Roussos

Max Planck Institute for Solar System Research, Katlenburg-Lindau, Germany 
the need to develop power sources suitable for the outer Solar System. The UP science case is based around exploring the origins, evolution, and processes at work in Ice Giant planetary systems. Three broad themes were identified: (1) Uranus as an Ice Giant, (2) An Ice Giant planetary system, and (3) An asymmetric magnetosphere. Due to the long interplanetary transfer from Earth to Uranus a significant cruise-phase science theme was also developed. The UP mission concept calls for the use of a Mars Express/Rosetta-type platform to launch on a Soyuz-Fregat in 2021 and entering into an eccentric polar orbit around Uranus in the 2036-2037 timeframe. The science payload has a strong heritage in Europe and beyond and requires no significant technology developments.

Keywords Uranus • Ice Giant • Orbiter • Giant planet atmosphere • Ring system $\cdot$ Interior $\cdot$ Dynamo $\cdot$ Magnetosphere $\cdot$ Natural satellite

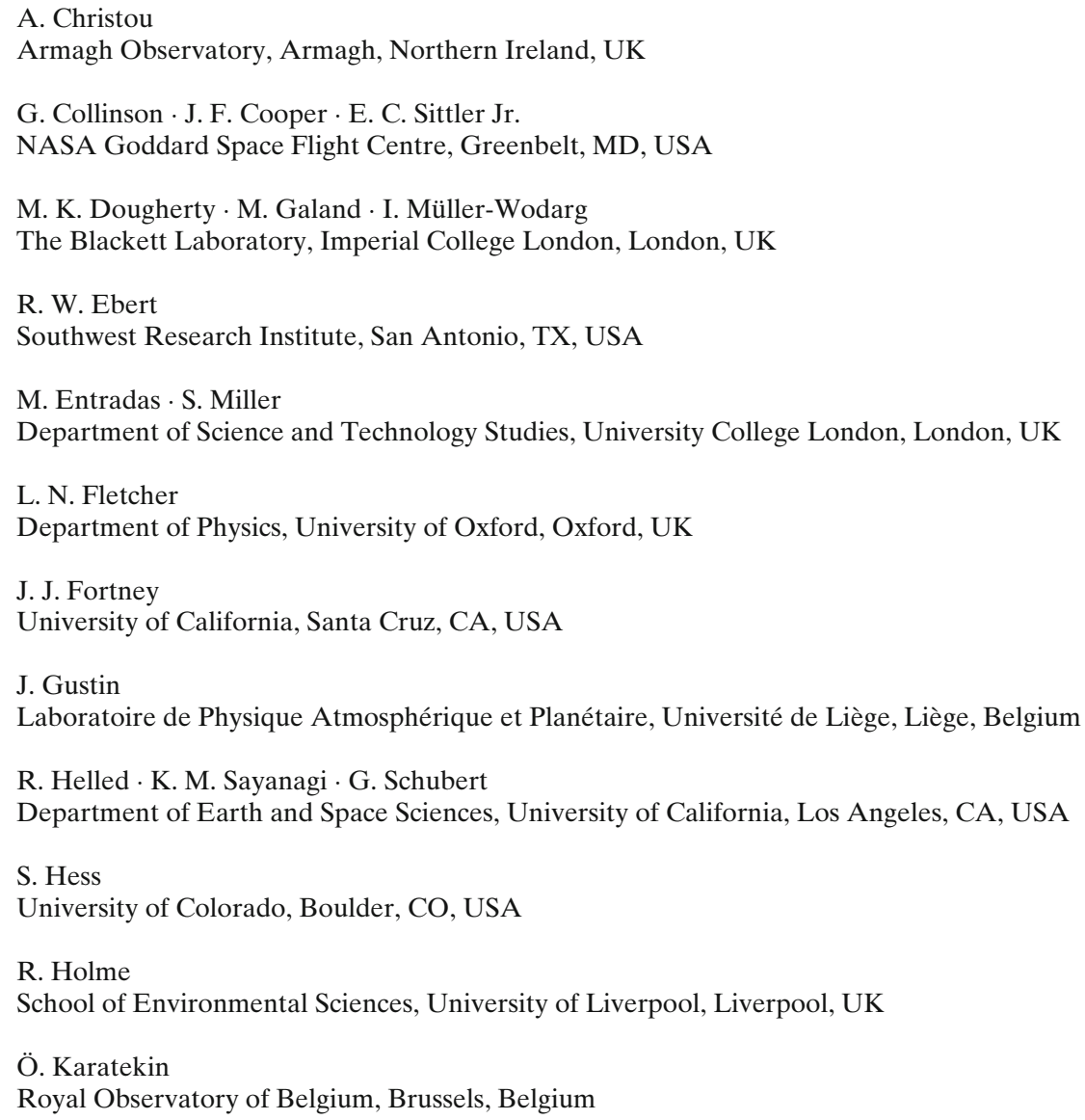




\section{Acronyms}

$\begin{array}{ll}\text { ALMA } & \text { Atacama Large Millimetre Array } \\ \text { AOCS } & \text { Attitude and Orbit Control } \\ \text { ASRG } & \text { Advanced Stirling Radioisotope Generator } \\ \text { CMI } & \text { Cyclotron Maser Instability } \\ \text { CSDS } & \text { Cluster Science Data System } \\ \text { E-ELT } & \text { European Extremely Large Telescope } \\ \text { EJSM } & \text { Europa Jupiter System Mission } \\ \text { ESA } & \text { European Space Agency } \\ \text { ESO } & \text { European Southern Observatory } \\ \text { ESOC } & \text { European Space Operations Centre } \\ \text { FUV } & \text { Far Ultraviolet } \\ \text { GTO } & \text { Geostationary Transfer Orbit } \\ \text { HGA } & \text { High Gain Antenna } \\ \text { IRTF } & \text { Infrared Telescope Facility } \\ \text { ISO } & \text { Infrared Space Observatory } \\ \text { JWST } & \text { James Webb Space Telescope }\end{array}$

S. Kemble $\cdot$ L. Peacocke

EADS Astrium, Stevenage, UK

Y. Langevin

Institut d'Astrophysique Spatiale, CNRS/Univ. Paris-Sud 11, Orsay, France

J. Leisner

Department of Physics and Astronomy, University of Iowa, Iowa City, IA, USA

J. Martin-Torres

Centre for Astrobiology, Madrid, Spain

T. Stallard $\cdot$ H. Melin

Department of Physics and Astronomy, University of Leicester, Leicester, UK

O. Mousis

Institut UTINAM, CNRS, OSU THETA, Université de Franche-Comté, Besançon, France

N. Nettelmann

Universität Rostock, Rostock, Germany

B. Noyelles

Namur Centre for Complex Systems (NAXYS), University of Namur, Namur, Belgium

I. de Pater

University of California, Berkeley, USA

M. Pätzold · S. Tellmann

Rhenish Institute for Environmental Research, University of Cologne, Cologne, Germany

E. Quémerais

LATMOS, CNRS, Guyancourt, France

包 Springer 


$\begin{array}{ll}\text { LILT } & \text { Low Intensity Low Temperature } \\ \text { MAG } & \text { Magnetometer (UP Instrument) } \\ \text { MMRTG } & \text { Multimission Radioisotope Thermal Generator } \\ \text { MWR } & \text { Microwave Radiometer (UP Instrument) } \\ \text { NAC } & \text { Narrow Angle Camera (UP Instrument) } \\ \text { NIR/MSIC } & \text { Visual and Near-Infrared Mapping Spectrometer and } \\ & \text { Multispectral Imaging Camera (UP Instrument) } \\ \text { ORS } & \text { Optical Remote Sensing } \\ \text { PDS } & \text { Planetary Data System } \\ \text { PI } & \text { Principal Investigator } \\ \text { PPS } & \text { Plasma and Particle Science (UP Instrument) } \\ \text { PSA } & \text { Planetary Science Archive } \\ \text { RPS } & \text { Radioactive Power Source } \\ \text { RPW } & \text { Radio and Plasma Wave Experiment (UP Instrument) } \\ \text { RSE } & \text { Radio Science Experiment (UP Instrument) } \\ \text { SSR } & \text { Solid State Recorder } \\ \text { TMT } & \text { Thirty Metre Telescope } \\ \text { TRL } & \text { Technology Readiness Level } \\ \text { UKR } & \text { Uranus Kilometric Radiation } \\ \text { UOI } & \text { Uranus Orbit Insertion } \\ \text { UP } & \text { Uranus Pathfinder }\end{array}$

C. T. Russell

Institute for Geophysics and Planetary Physics, University of California Los Angeles, Los Angeles, USA

A. Sánchez-Lavega

University of the Basque Country, Bilbao, Spain

J. Saur

Institute of Geophysics and Meteorology, University of Cologne, Cologne, Germany

P. Schenk

Lunar and Planetary Institute, Houston, TX, USA

N. Sergis

Office for Space Research, Academy of Athens, Athens, Greece

F. Sohl

Institute of Planetary Research, DLR, Berlin, Germany

N. A. Teanby

School of Earth Sciences, University of Bristol, Bristol, UK

G. Tobie

LPG, CNRS, Université de Nantes, Nantes, France

J.-E. Wahlund

Swedish Institute of Space Physics, Uppsala, Sweden 


$\begin{array}{ll}\text { UPMOC } & \text { Uranus Pathfinder Mission Operations Centre } \\ \text { UPQL } & \text { Uranus Pathfinder Quicklook } \\ \text { UPSOC } & \text { Uranus Pathfinder Science Operations Centre } \\ \text { UTIRM } & \text { Thermal Infrared Bolometer (UP Instrument) } \\ \text { UVIS } & \text { Ultraviolet Imaging Spectrometer (UP Instrument) } \\ \text { VLA } & \text { Very Large Array } \\ \text { VLT } & \text { Very Large Telescope } \\ \text { WFIRST } & \text { Wide-Field Infrared Survey Telescope }\end{array}$

\section{Introduction}

The canonical architecture of the Solar System often groups the Gas Giant planets, Jupiter and Saturn, together with the Ice Giants, Uranus and Neptune, and refers to them as the giant planets. However, the importance of volatile materials (known as ices) such as methane in the interiors and atmospheres of Uranus and Neptune, the highly asymmetric configuration of their magnetic fields, and their different internal structure (amongst other things) clearly distinguish the Ice Giants as a very different class of planet. In order to unravel the origin and evolution of the Solar System one must understand all of its components. In this regard Uranus and Neptune are enigmatic objects with very poorly constrained interiors, magnetic fields, atmospheres, ring and satellite systems and magnetospheres, among just a few of the intriguing aspects of these systems. The importance of filling these gaps in our knowledge of the Solar System is particularly acute when trying to apply our understanding to the numerous planetary systems that have been discovered around other stars.

Uranus occupies a unique place in the history of the Solar System and the fundamental processes occurring within the uranian system confirm that its scientific exploration is essential in meeting ESA's Cosmic Vision goals (see Section 2, particularly Section 2.4 and Table 2). Table 1 illustrates the key properties of the uranian system. Uranus Pathfinder (UP) was proposed to the European Space Agency's Cosmic Vision 2015-2025 call for medium "M"

Table 1 Physical and orbital parameters of Uranus

\footnotetext{
a Seidelmann et al. [48]

${ }^{b}$ Jacobson et al. [36]

${ }^{\mathrm{c}}$ Desch et al. [14]

dNASA HORIZONS system: http://ssd.jpl.nasa.gov/

?horizons

${ }^{\mathrm{e}}$ Herbert [31]

${ }^{\mathrm{f}}$ http://ssd.jpl.nasa.gov/

?sat_discovery (Accessed 29

July 2011)
}

\begin{tabular}{|c|c|}
\hline $\begin{array}{l}\text { Equatorial radius } \\
\text { Polar radius }^{\mathrm{a}}\end{array}$ & $\begin{array}{l}25559 \pm 4 \mathrm{~km}\left(=1 \mathrm{R}_{\mathrm{U}}\right) \\
24973 \pm 20 \mathrm{~km}\end{array}$ \\
\hline Mass $^{b}$ & $14.5 \mathrm{M}_{\mathrm{E}}\left(1 \mathrm{M}_{\mathrm{E}}=5.97 \times 10^{24} \mathrm{~kg}\right)$ \\
\hline Rotation period ${ }^{\mathrm{c}}$ & $17 \mathrm{~h} 14 \mathrm{~m} 24 \mathrm{~s}( \pm 36 \mathrm{~s})$ \\
\hline Obliquity $^{\mathrm{a}}$ & $97.77^{\circ}$ \\
\hline Semi-major axis ${ }^{\mathrm{d}}$ & $19.2 \mathrm{AU}$ \\
\hline Orbital period ${ }^{\mathrm{d}}$ & 84.3 Earth years \\
\hline Dipole moment $\mathrm{e}^{\mathrm{e}}$ & $3.75 \times 10^{24} \mathrm{~A} \mathrm{~m}^{2}$ \\
\hline Magnetic field strength ${ }^{\mathrm{e}}$ & Max: $1.0 \times 10^{5}$ \\
\hline (in uranographic equator) & Min: $7.7 \times 10^{3} \mathrm{nT}$ \\
\hline Dipole tilt $\mathrm{e}^{\mathrm{i}}$ & $-59.8^{\circ}$ \\
\hline Dipole offset ${ }^{\mathrm{e}}$ & $0.31 \mathrm{R}_{\mathrm{U}}$ (southward) \\
\hline Natural satellites ${ }^{\mathrm{f}}$ & 27 (9 irregular) \\
\hline
\end{tabular}


class missions in 2010. The mission concept called for the first orbiter of an Ice Giant and would open a new window on the origin and evolution of the Solar System, and the fundamental physical processes at work at giant planets. UP thus embodies the quintessential aspects of ESA's Cosmic Vision 2015-2025 providing important information on the origin and evolution of Uranus as the archetypal Ice Giant representing the missing link between our Solar System and planets around other stars.

The importance of an Ice Giant mission was highlighted in the 2011 NRC Planetary Science Decadal Survey [54] where it was noted "A mission combining an orbiter and a probe will revolutionize our understanding of Ice Giant properties and processes, yielding significant insight into their evolutionary history". Although Neptune and its large satellite Triton are very interesting Solar System targets, Squyres et al. [54] note that risks associated with aerocapture at Neptune, the lack of optimal launch windows for Neptune over the coming decade, and long transfer times render a Uranus mission more attractive in the 2013-2023 time frame. The science priorities for a Uranus orbiter described by Squyres et al. [54] are similar to those for Uranus Pathfinder thus demonstrating considerable international consensus regarding the science goals and scientific return for such an orbiter. A mission to Uranus was discussed as a promising future mission in the previous decadal survey [3].

The scientific goals of UP are centred on three key scientific themes: (1) Uranus as an Ice Giant; (2) Uranus and its environment: An Ice Giant planetary system; and (3) A distinctively asymmetric magnetosphere. Due to the long transfer time from Earth to Uranus, the UP mission concept also calls for a significant cruise phase science programme involving flybys of small Solar System objects and answering fundamental questions about the transport of mass, energy and momentum from the Sun out into the heliosphere. In addressing these four themes (three prime science plus cruise phase) UP directly addresses two of the Cosmic Vision 2015-2025 themes "What are the conditions for Planet Formation and the Emergence of Life?" and "How Does the Solar System Work?".

The UP mission concept is novel in attempting to explore such a distant Solar System target within the M class cost cap of $470 \mathrm{M} €$ (FY 2010). The nominal UP mission involves a launch on a Soyuz-Fregat launch vehicle in 2021 with a $\approx 15$-year cruise before entering into a highly elliptical science orbit around Uranus. To reduce cruise phase costs UP would be placed into a quasihibernation mode, similar to Rosetta [26] and New Horizons [21], and would make solar wind measurements en route to Uranus. UP would periodically come out of hibernation to downlink solar wind science data and spacecraft telemetry to Earth. The science payload has strong heritage within Europe and beyond and takes advantage of the latest in low-mass science instrumentation. With current technology a solely solar-powered mission to Uranus would be prohibitively expensive and challenging so as part of the mission concept development we have investigated radioisotope power sources (RPSs). The UP proposal shows that significant scientific missions can be carried out using RPSs that employ isotopes other than ${ }^{238} \mathrm{Pu}$. The baseline RPS devices are 
based around ${ }^{241} \mathrm{Am}$ which, as a waste product from the nuclear reactors, is readily available within Europe.

The mission has significant community support within Europe and worldwide as reflected by (i) the 169 scientists across the world (105 in Europe) lending their support to the mission; (ii) the key planetary objectives specified by numerous Uranus-related white paper submissions to NASA's Planetary and Heliophysical Decadal Surveys; and (iii) NASA's formal recognition of the relevance of Uranus Pathfinder for addressing key planetary science goals. Perhaps unsurprisingly, the level of community support is highest among earlyand mid-career scientists, although there is significant support from senior levels of the community, many of who were involved in the Voyager 2 encounter with Uranus. More details on the UP mission concept and community can be found at http://bit.ly/UranusPathfinder.

\section{Scientific objectives}

The overarching theme for UP is the exploration of the origin, evolution and processes at work in Ice Giant planetary systems. Uranus is the centre of one of the Solar System's most interesting planetary systems and UP will study the fundamental processes at work on the planet itself (its interior and atmosphere) and in its planetary environment (magnetosphere, satellites and rings). The mission will provide observations and measurements that are vital for understanding the origin and evolution of Uranus as an Ice Giant planet, providing a missing link between our Solar System and planets around other stars. UP thus represents the quintessential aspects of the objectives of the European planetary community as expressed in ESA's Cosmic Vision 20152025.

Ice Giant planets account for more than 7\% of the mass of the Sun's planetary system, and helped to shape the conditions we see in the Solar System today. The number of Uranus-sized extrasolar planets discovered to date, weighted by the likelihood of observing them, indicates that such planets are common in the Universe. The Ice Giants are fundamentally different from the Gas Giants (Jupiter and Saturn) in a number of ways and yet our exploration of the Ice Giants in our own Solar System remains very incomplete, with a significant number of fundamental questions unanswered. The earliest possible date for the arrival of a new spacecraft mission at Uranus (not necessarily UP) leaves a >40 year gap since the flyby of Voyager 2 in 1986 (e.g., [58], and references therein; [4, 59], and other articles in J. Geophys. Res. Volume 92 Issue A13 pages 14873-15375) and underlines the urgent need for new measurements. UP will provide new insights into the formation, bulk composition, and evolution of Uranus-mass objects in our Solar System and beyond. The measurements of atmospheric composition, structure and dynamics by UP will be of enormous value for interpreting telescopic observations of many exoplanets. Understanding the magnetosphere and radio emissions of Uranus will also be of immense value in understanding exoplanet magnetospheres. 


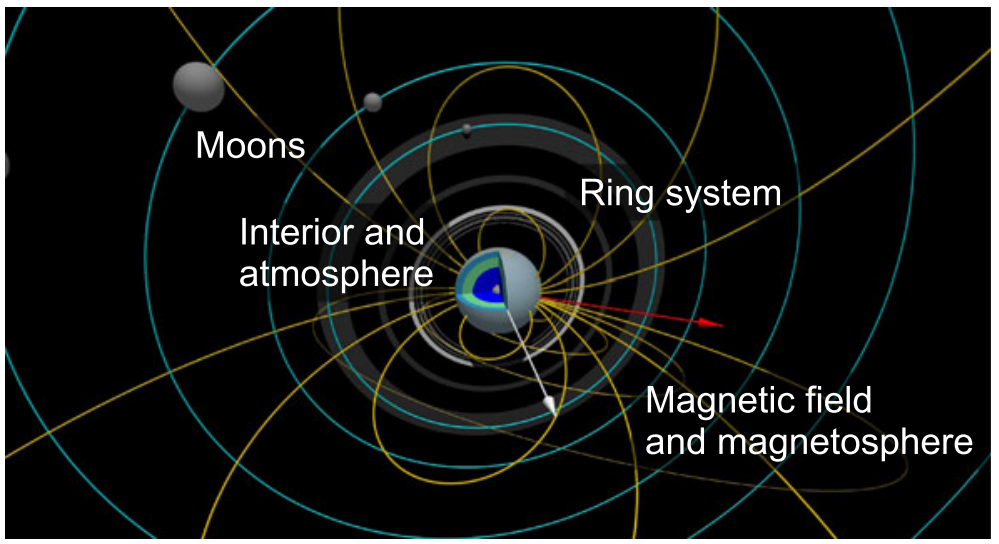

Fig. 1 Illustration showing the rich variety of science goals for the UP mission: variety of natural satellites, complex ring system, highly asymmetric magnetic field and magnetosphere, atmosphere and interior. The white arrow indicates the spin axis of Uranus whereas the red arrow indicates the magnetic dipole axis. The orbits of the five major satellites are shown in blue with magnetic field lines in yellow

Figure 1 illustrates the rich variety of science goals for the UP. This illustration is drawn from the perspective of the Sun during the Voyage 2 encounter and highlights one of the unique aspects of Uranus: it's large $98^{\circ}$ obliquity.

The prime scientific goals for UP are built upon three themes: (1) Uranus as an Ice Giant; (2) An Ice Giant planetary system; (3) An asymmetric magnetosphere. To focus this mission description on the prime science phase, the fourth theme consisting of cruise phase science will not be discussed here.

\subsection{Uranus as an Ice Giant}

The bulk composition and internal structure of the Ice Giants reflect their different formation environments and evolutionary processes relative to the Gas Giants (e.g. [27]) providing a window into the early Solar System. Jupiter is an $\mathrm{H} / \mathrm{He}$ planet with an ice and rock mass fraction of $4-12 \%$ as inferred from standard interior models [45]. Uranus and Neptune seem to consist mostly of "ices" $\left(\mathrm{H}_{2} \mathrm{O}, \mathrm{NH}_{3}, \mathrm{CH}_{4}\right)$ and rocks, with smaller envelopes of $\mathrm{H}_{2}$ and $\mathrm{He}$, but current observations are only able to provide an upper limit of $85 \%$ on the ice and rock mass fraction [19].

The Nice model, proposed to explain the origin of the orbital parameters of the giant planets [65] predicts that Uranus formed closer to the Sun than its present location (but possibly further out than Neptune) and then migrated outwards (along with Neptune) scattering planetesimals and triggering the late heavy bombardment of the inner solar system. The composition of Uranus contains clues to the conditions in the protosolar cloud and the locations in which the planet formed, thus providing vital inputs to the further study of migration and the mass in the protosolar cloud (e.g., [9]). For instance, a sub-solar 
C:O ratio could indicate formation at a distance where water (but not $\mathrm{CH}_{4}$ ) was frozen. The common picture of gaseous planet formation by first forming a $10 \mathrm{M}_{\mathrm{E}}$ core $\left(1 \mathrm{M}_{\mathrm{E}}=5.97 \times 10^{24} \mathrm{~kg}\right)$ and then accreting a gaseous envelope is challenged by state-of-the-art interior models, which instead predict rock core masses below $5 \mathrm{M}_{\mathrm{E}}[19,45]$. Uranus' obliquity and low heat loss may point to a catastrophic event and provides additional important constraints for planetary system formation theories (also see [5]).

The composition of the uranian atmosphere from remote sensing and/or in situ probing (elemental enrichments, isotopic ratios and noble gases) can be extrapolated to provide important clues about the bulk composition of the deep interior, and provides a window onto conditions in the solar nebula during the era of planetary formation. UP will reveal the fundamental processes that shape the formation, evolution, dynamic circulation and chemistry of Ice Giant atmospheres. There is currently no interior model for Uranus that agrees with all the observations, representing a significant gap in our understanding of the Solar System (see Fig. 2a for one such model). To develop improved models of Uranus' interior better compositional data must be obtained [30]. Understanding the internal structure of Uranus (the nearest Ice Giant) is essential for estimating the bulk composition of the outer planets, in particular their ice-to-rock ratio.

Planet interiors are initially warm and cool down as they age. Gravitational energy from material accretion is converted to intrinsic, thermal energy during formation and is steadily radiated away through their atmospheres. Thermal evolution models probe the energy reservoir of a planet by predicting its intrinsic luminosity. Such models reproduce the observed luminosity of Jupiter and Neptune after $4.56 \mathrm{Ga}$ of cooling, independent of detailed assumptions

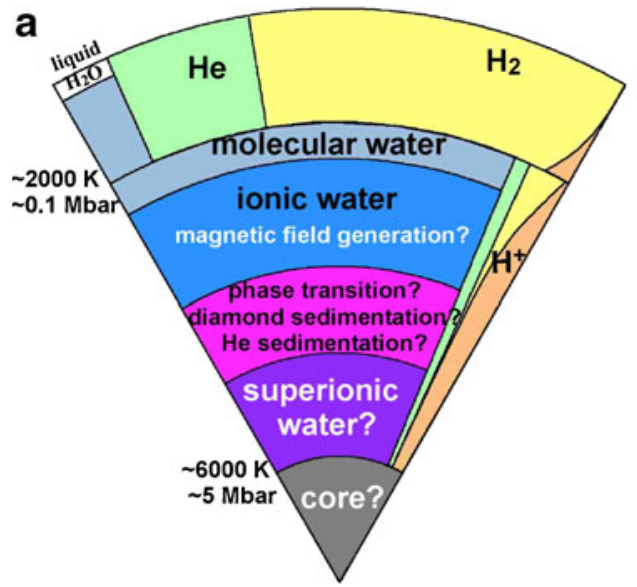

b

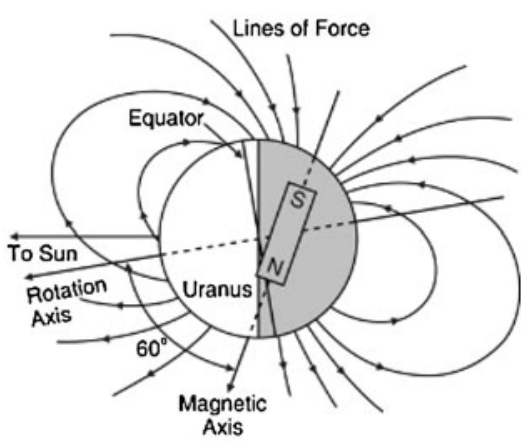

Fig. 2 Illustrations showing a model of Uranus' interior that is consistent with the gravity and magnetic field data but not with Uranus' low luminosity (Nettelmann, private communication); b the configuration of Uranus' internal magnetic field [41] 
about their atmosphere, albedo, and solar irradiation. The same models, however underestimate Saturn's luminosity and overestimate it for Uranus [20]. Indeed, Uranus' is so cold and its intrinsic luminosity is so low that, according to standard thermal evolution theory, Uranus should be more than 3 billion years older than it is (where the observational uncertainty in luminosity accounts for about 2 billion years). The intrinsic luminosity of Uranus [43] also has implications for understanding planetary dynamos and magnetic field generation. The unusual, but poorly constrained [33], configuration of Uranus' intrinsic magnetic field (see Fig. 2b) suggests some fundamental difference between the dynamos of Uranus and Neptune and those of the other planets $[55,56]$. The field is also expected to have undergone secular change since the Voyager 2 epoch [8].

The small envelopes of $\mathrm{H}_{2}-\mathrm{He}$ and substantial enrichment of heavy elements in the Ice Giants, the cold atmospheric temperatures relative to the Gas Giants (Jupiter and Saturn), and the extreme obliquity yield unique physicochemical conditions that cannot be found elsewhere in the Solar System. Uranus therefore provides an extreme test of our understanding of many aspects of planetary atmospheres, including: dynamics, energy and material transport, seasonally varying chemistry and cloud microphysics, and structure and vertical coupling throughout giant planet atmospheres. Uranus' weather layer (the troposphere and lower stratosphere) can be studied via infrared, sub-millimetre and microwave remote sensing (see Fig. 3) to reveal the atmospheric temperature structure, gaseous composition and distribution of cloud opacity. These parameters can be used to trace the dynamics, circulation

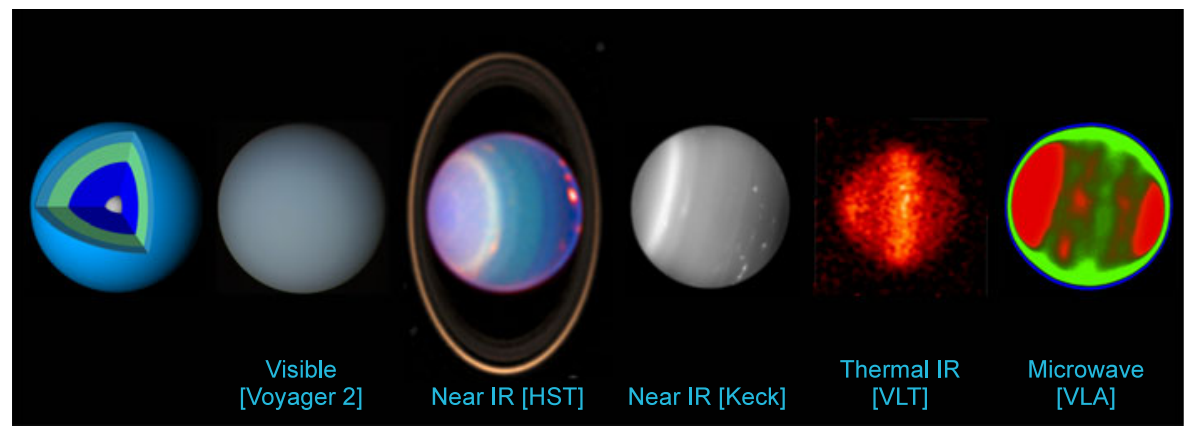

Fig. 3 Model of Uranus' interior compared with Uranus' appearance at multiple wavelengths, sensitive to reflection and scattering of reflected sunlight from uranian clouds and aerosols (first three are short-wavelength images from Voyager 2 (a), HST (b) and Keck (c)), and to thermal emission from atmospheric gases at longer wavelengths (last two images from the VLA (d) and VLT (e)). Although Uranus appeared relatively tranquil in images obtained by Voyager 2 due to obscuring tropospheric hazes, multi-wavelength imaging at longer wavelengths demonstrate the wide range of discrete cloud activity and the distributions of gaseous opacity sources on the Ice Giant. Credits: (a) NASA/JPL; (b) E. Karkoschka (University of Arizona, USA), Hubble Space Telescope and NASA; (c) H. Hammel (Space Science Institute, Boulder, USA), I. de Pater (University of California Berkeley, USA), W.M. Keck Observatory; (d) G. Orton (NASA JPL); (e) M. Hofstadter (NASA JPL) 
and chemistry of the weather layer, both in terms of small-scale convective events (storms, plumes and vortices, like the discrete activity in Fig. 3 and planetary-scale circulation. Unlike the Gas Giants, Uranus exhibits a strong westward jet at its equator and seasonally variable circumpolar collars. Vertical sounding in the troposphere and stratosphere, as well as cloud tracking and the monitoring of dynamical tracers (e.g., hydrocarbons, condensable volatiles, disequilibrium species and microwave opacity sources) are essential to explain the stark differences in energy and material transport on gas and Ice Giants. Finally, the spatiotemporal mapping of stratospheric hydrocarbons and oxygenated species would reveal (a) the rich variety of photochemical pathways at work at 19.2 AU, and (b) the sources and variability of exogenic materials (from meteoritic bombardment or other impact processes) to understand the connection between an Ice Giant atmosphere and its immediate planetary environment (theme 2). Methane is the prime condensable which forms clouds in the upper troposphere (near the 1 bar level) while a number of hydrocarbons (e.g., acetylene and ethane) can form hazes in the stratosphere $(<100 \mathrm{mbar}$ level). Although it is not understood why the methane clouds are sparse and thin when methane comprises $\sim 15 \%$ of the atmospheric mass.

On Jupiter and Saturn, two end-point scenarios have been suggested as the forcing mechanism for the jets: (1) deep internal convection driven by internal heat flux, and (2) shallow turbulence in the surface "weather" layer driven by thunderstorms and solar heating (see review in [66]). The observed low internal heat flux from Uranus and low occurrence of atmospheric turbulence raises questions about the contributions from both of these mechanisms. However, under the influence of strong rotation, turbulence has been shown to generate and maintain jetstreams by, for example, Showman [52] and Sayanagi et al. [47], i.e., large-scale turbulence acts in pumping the jets rather than dissipating them. Thus, the apparent lack of turbulence in Uranus' atmosphere argues for a comparative study against the fully turbulent atmospheres of Jupiter and Saturn. Uranus Pathfinder's high-resolution atmospheric imaging campaign will seek the turbulent processes that force the wind system. Great dark spots have recently been observed on Uranus [28] and turbulence in the form of small-scale eddies may also be involved in their formation, however, a complete theory is not yet available. Observations of Uranus' atmosphere is crucial for understanding the energy and momentum cycle that powers jetstreams and large vortices in Ice Giant atmospheres.

On the other hand, the temperature in Uranus' upper atmosphere (thermosphere and ionosphere) is several hundred degrees hotter than can be explained by solar heating. Moreover, this temperature is strongly correlated with season such that the upper atmosphere is more than $200 \mathrm{~K}$ hotter at solstice than at equinox [39]. Since the southern hemisphere was almost continually illuminated at solstice, the influence of the Sun must have a strong part to play in explaining the considerable temperature excess beyond the heating that the Sun can provide directly. The thermosphere and ionosphere form a crucial transition region between interplanetary space and the planet itself. Powerful currents, generated by electric fields imposed by the 
magnetosphere of magnetised planets, may result in large energy inputs to the upper atmosphere due to Joule heating and ion drag. The energy from these sources may be tens to hundreds of times greater than that due to the absorption of solar extreme ultraviolet radiation. It seems likely that a key component of the required additional heating is driven by particle precipitation and/or the way in which varying magnetospheric configurations couple with the upper atmosphere to produce time-variable fields and currents. Magnetospheric particle precipitation driven by corotating plasma interactions with the moons and rings of Uranus may also play a role in the atmospheric energy budget. A similar excess temperature is also found in the saturnian and jovian upper atmospheres. Thus, this "energy crisis" is a fundamental problem in our general understanding of the workings of giant planet upper atmospheres. A mission to Uranus' unusually asymmetric magnetosphere provides an opportunity to understand how insolation and particle precipitation from the solar wind and magnetosphere contribute to the energy balance in the upper atmosphere.

\subsection{An Ice Giant planetary system}

Uranus has a rich planetary system of both dusty and dense narrow rings and regular and irregular natural satellites. This unique example of a planetary system holds important information to help us unravel the origin and evolution of the Solar System. Ground-based observations have found changes in the rings and satellites since the Voyager 2 flyby indicating fundamental instabilities in the coupled ring-moon system [50] of clear importance for understanding the evolution of planetary systems. Study of the moons and rings of Uranus, in particular their composition and dynamical stability, the internal and subsurface structure of the moons, and the geological history of the moons (and how that relates to their formation) is important for understanding how the Solar System formed and evolved. The possibility that Uranus' irregular satellites are captured Centaurs, trans-neptunian objects, or comets (e.g., [13]) would also contribute to understanding small Solar System bodies and may provide lessons for our understanding of the origin of life in the Solar System, particularly since objects exposed to the solar wind are subjected to very different space weathering processes than those protected from the solar wind within Uranus' magnetosphere.

\subsubsection{Ring system}

The composition of the ring system provides significant constraints on planetary evolution models. Unfortunately, Voyager could not detect them in the infrared (the important wavelength range for ring composition) and so the composition of the rings is essentially unknown. The particle-size distribution of Uranus' main rings was studied from Voyager 2 radio occultations but detected a surprising lack of centimeter-size particles [22]. High spatial resolution imaging of the narrow rings is needed to unravel the dynamics of their 
Fig. 4 This composite image of Uranus' main rings in forward-scattered (left) and back-scattered (right) light shows that a network of dust structures is interleaved among the planet's dense main rings. The offset in the $\varepsilon$ ring is due to its eccentricity. As the left-hand image is the only high-phase image ever taken of Uranus' rings (by the post-encounter Voyager 2), the detailed workings of the dust structures remain largely unknown. Credit: NASA/JPL

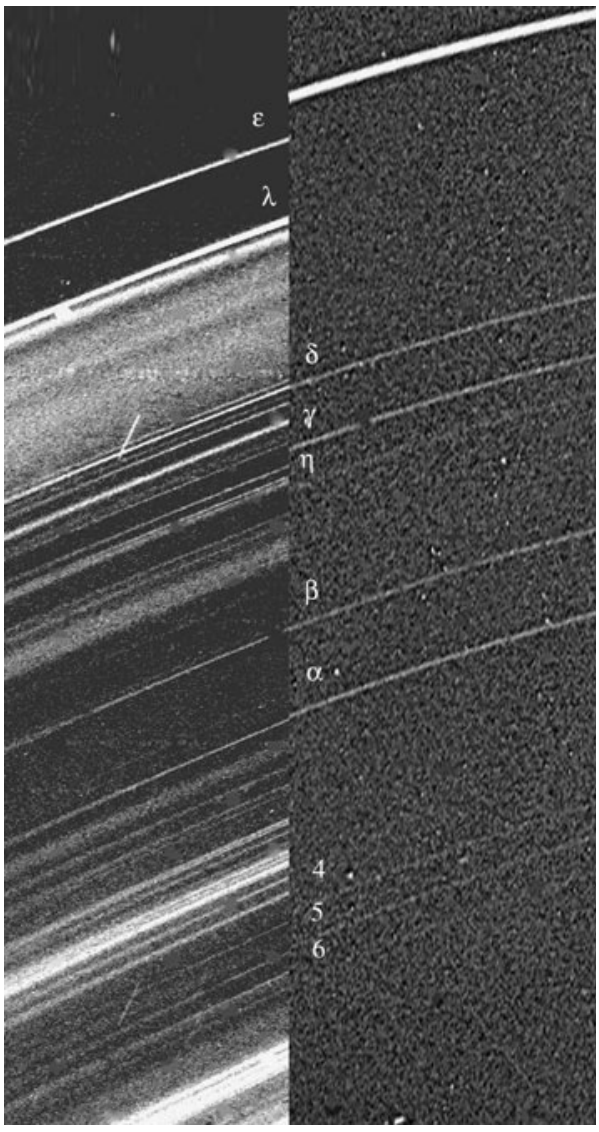

confinement and to confirm theories of self-maintenance and of shepherding by moons, which are relevant to other disk systems including protoplanetary disks (e.g., [16, 17, 22, 50]). The dusty rings also present challenges for existing theories [40]. Voyager's single high-phase image of the rings revealed a plethora of otherwise unknown dust structures (Fig. 4). Since the Voyager encounter in 1986, large-scale changes have been discovered in these rings, such as the apparent "displacement" (or disappearance and creation of) the innermost zeta ring [12]. Of particular interest is the newly discovered mu ring at $\sim 4 \mathrm{R}_{\mathrm{U}}$, which appears to be as blue as Saturn's E ring [11, 50]. More details of the structure of the rings and a first understanding of their evolution would be immensely valuable. Also of interest are the rings' interactions with Uranus' extended exosphere and their accretion/disruption interplay with the nearby retinue of small moons $[11,16,50,51]$.

\subsubsection{Natural satellites}

Uranus' five largest moons (Miranda, Ariel, Umbriel, Titania, Oberon-see Fig. 5) are comparable in size to the medium-sized moons of Saturn, although 


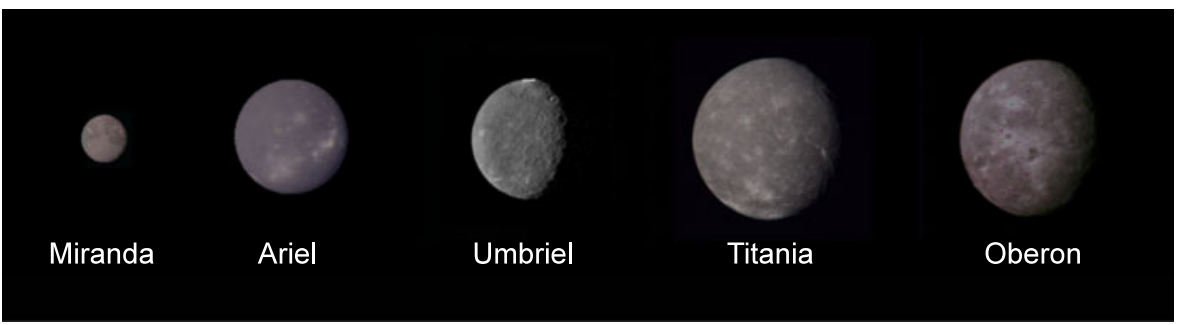

Fig. 5 Voyager 2 images of the five largest moons of Uranus. Voyager passed closest to the innermost of these satellites and so the imaging resolution is best at Miranda, while Titania and Oberon were not imaged at sufficiently high resolution to resolve details of tectonic structures (Credit: Paul Schenk/NASA/JPL)

their mean densities $\left(\approx 1,500 \mathrm{~kg} \mathrm{~m}^{-3}\right.$, on average) are higher. The moons also have similar orbital configurations to those at Saturn, but Uranus' large obliquity results in significantly different insolation patterns, with one pole directed towards the sun during solstice. The observations performed during the flyby of Voyager 2 revealed signs of endogenic resurfacing, particularly on Miranda and Ariel, associated with tectonic systems and possibly involving cryovolcanic processes. As in the jovian and saturnian systems, tidal and magnetospheric interactions are likely to have played a key role in the evolution of the uranian satellite system. For instance, intense tidal heating during sporadic passages through orbital resonances is expected to have induced internal melting in some of the icy moons [61, 62]. One such tidally-induced melting event may have triggered the geological activity that led to the late resurfacing of Ariel. The two largest moons, Titania and Oberon have diameters exceeding $1,500 \mathrm{~km}$ and past melting events may have left liquid water oceans beneath their outer ice shells (e.g. [35]). The strongly inclined magnetic dipole moment of Uranus with respect to its spin axis generates time-variable fields near the moons at their synodic rotation periods. These fields will produce induction magnetic fields, which are diagnostic of the moons interior, in particularly with respect to the possible salty liquid sub-surface oceans in Titania and Oberon [46]. The tilt of the magnetic equatorial plane with respect to the ring plane also means that magnetospheric plasma and energetic particle irradiation of the moons varies strongly in time, peaking periodically as the moons cross the magnetic equator [57]. This pulsed mode of irradiation may have impacts on chemical evolution and transient activity of the moon surfaces and may be remotely detectable in terms of ultraviolet, X-ray, gamma, and energetic neutral atom emissions.

As the main natural satellites in the system, these five moons are assumed to be locked in the Cassini State 1, consisting of the spin-orbit 1:1 resonance and an equilibrium obliquity (e.g., [25, 48]). Departures from this Cassini State would give indications on the internal structure of the satellites, as proposed by Peale et al. [42] for Mercury. Moreover, a measure of their rotation frequency could reveal an internal ocean, as it is the case for Titan [37] and Europa 
[23]. In the case of Miranda, a signature of the recent disruption of an orbital resonance forcing its inclination [60] could be seen in its obliquity.

Understanding the geologic evolution and tectonic processes of the five major satellites of Uranus suffers from incomplete Voyager imaging. Coverage was restricted to the southern hemispheres and the medium to low image resolutions (order of several kilometres per pixel, except for part of Miranda) only allow a limited characterisation of the major geologic units in the areas imaged by Voyager (e.g., [10]). The crater size-frequency distributions of the five satellites, used as a tool for age-dating of surface features and for assessing projectile populations and origins thereof, are known only for the southern hemispheres and at crater sizes larger than a few kilometres (e.g. [44]). The bulk composition of the moons are fundamentally important in constraining the origin of these bodies, although large uncertainties still exist on these parameters (e.g. [35]). The diversity of the medium-sized icy satellites in the uranian system demonstrates the complex and varied histories experienced by this class of object.

UP will reveal the nearly-unexplored uranian satellites by observing their Northern Hemispheres for the first time and by constructing extensive, multi-wavelength maps of the moons and rings that were not possible with Voyager 2.

\subsection{The asymmetric magnetosphere}

The configuration of each planetary magnetosphere in the Solar System is determined by the relative orientations of the planet's spin axis, its magnetic dipole axis and the solar wind flow. In the general case, the angle between the magnetic dipole axis and the solar wind flow is a time-dependent quantity and varies on both diurnal and seasonal timescales. Uranus presents a particularly special and poorly-understood case because this angle not only varies seasonally but because of Uranus' large obliquity the extent of diurnal oscillation varies with season. At solstice this angle does not vary much with time and Uranus' magnetic dipole simply rotates around the solar wind flow vector. This magnetospheric configuration is not found anywhere else in the Solar System. These significant asymmetries produce large-scale diurnal reconfigurations of the system on timescales of hours resulting in a twisted magnetotail topology $[2,63]$. The near alignment of the rotation axis with the planet-Sun line during solstice means that plasma motions produced by the rotation of the planet and by the solar wind are effectively decoupled [49, 67]. In Sittler et al. [53] from Voyager 2 plasma observations they showed evidence for injection events to the inner magnetosphere likely driven by reconnection events every planetary rotation period when the dipole field is oppositely directed to the interplanetary field. Therefore, in contrast to Jupiter and Saturn, solar wind plasma may be able to penetrate deep within the magnetosphere despite the planet being a fast oblique rotator.

Because of this unique extreme orientation, Uranus' magnetosphere varies from a pole-on to orthogonal configuration during a uranian year (84 Earth 
years) and changes from an "open" to a "closed" configuration during a uranian day. Such a rapidly reconfiguring magnetosphere with a highly asymmetric internal magnetic field at its core provides a challenge for current theories of how magnetospheres work. The UP mission, on-orbit for many months will bring new insights into understanding universal magnetospheric processes. Uranus also presents a special case because of its distant location in the heliosphere where the properties of the solar wind are very different from the other planets we've explored in detail. This provides opportunities to investigate fundamental processes such as magnetic reconnection and collisionless shocks under different parameter regimes and to extend our understanding of space weather.

These aspects make a study of Uranus' magnetosphere-particularly close to solstice near the orbit insertion date of UP-a very important objective for understand how the Solar System works. They are not only essential in helping to understand how asymmetric Ice Giant magnetospheres work, but are also highly relevant in providing "ground-truth" for understanding exoplanet magnetospheres. UP will bring crucial constraints and fresh insights into how magnetospheres work and will fill the urgent need for new understanding to place the recent surge of exoplanet observations into context.

Along with the planetary magnetic field, the ionosphere of Uranus is the internal core of the magnetosphere. Models indicate that Uranus' ionosphere is dominated by $\mathrm{H}^{+}$at higher altitudes and $\mathrm{H}_{3}^{+}$lower down $[6,7,38]$, produced by either energetic particle precipitation or solar ultraviolet (UV) radiation. There has only been one spatially resolved observation of the UV aurora of Uranus [31], using a mosaic of Voyager 2 Ultraviolet Spectrograph (UVS) observations which mapped emission from $\mathrm{H}$ Lyman- $\alpha$ and the EUV $\mathrm{H}_{2}$ band (Fig. 6, left). The emission appears patchy and is generally centred on the magnetic poles, being the brightest about midnight magnetic local time. There have been subsequent attempts to observe the aurora both in the far ultraviolet (FUV) using the Hubble Space Telescope [1] and in the IR using ground-based

a

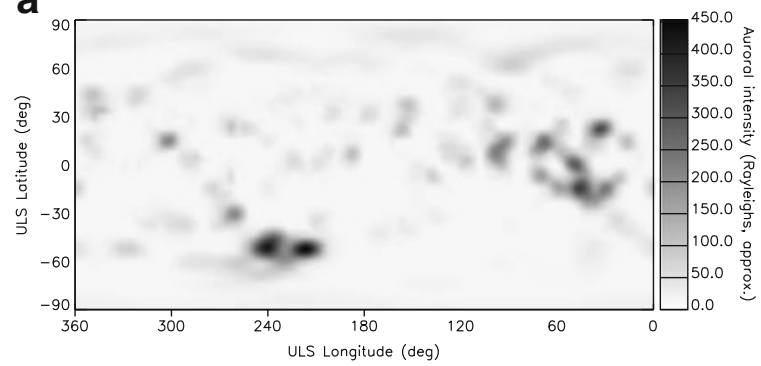

b

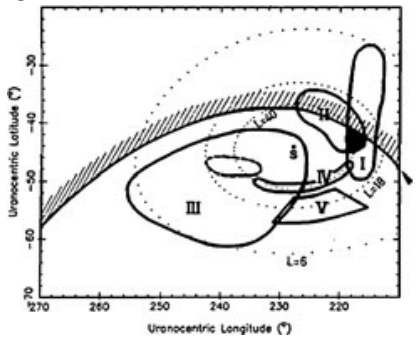

Fig. 6 (Left) $\mathrm{H}_{2}$ band emission map showing auroral intensity, ranging between 0 and 450 Rayleighs, for both aurorae, overplotted on the mapped magnetospheric distances from the planet as L-shells in steps of two [31]. (Right) Source regions inferred for the most intense UKR component [68] 
telescopes (e.g., [64]) but any spatially resolvable auroral features remain undetected. Recent analysis of observations of $\mathrm{H}_{3}^{+}$emissions from Uranus spanning almost 20 years [39] have revealed a phenomenon that is not seen at the other Gas Giants in our Solar System. As noted earlier, the temperature is strongly correlated with season, e.g., the upper atmosphere is more than $200 \mathrm{~K}$ hotter at solstice than at equinox. It seems likely that a key component of the required additional heating is driven by particle precipitation and/or the way in which varying magnetospheric configurations couple with the upper atmosphere.

Auroral emissions are also generated above the ionosphere at kilometric (radio) wavelengths $(1-1,000 \mathrm{kHz})$ (known as Uranus Kilometric RadiationUKR) which cannot be observed from Earth or by distant observers. Although the UKR emissions from the south pole are more intense than those from the north pole, the opposite was found to be true for emission in the $\mathrm{H}_{2}$ band from the aurora [32]. As at other planets, UKR is thought to be generated by the Cyclotron Maser Instability (CMI) around the magnetic poles and therefore is a remote marker of planetary rotation. UKR displays a rich variety of components characteristic of Ice Giants (see Fig. 6, right), including unique features such as time-stationary radio sources (e.g. [15], and references therein, and [69]).

Understanding the circumstances under which these radio emissions are generated is of prime importance for using them to the detection of exoplanetary magnetic fields (important for the development and protection of life). Unlike our Solar System, eccentric and complex orbital characteristics appear to be common in other planetary systems, so that the understanding of radio emission produced by Uranus could have profound importance for interpreting future radio detections of exoplanets (e.g. [70]).

\subsection{Summary: the scientific case for Uranus Pathfinder}

In summary, there are significant and unexplained differences among Ice Giant, terrestrial, and Gas Giant planetary systems that point to very different formation and evolutionary histories. With its highly asymmetric magnetic field, large obliquity, and unusually low amount of emitted internal heat, Uranus is the Ice Giant that differs most from the other planets and provides several extreme tests of our understanding of planetary interiors, atmospheres, magnetospheres, rings and satellites. The interior, atmosphere, magnetosphere and planetary environment will be studied as one three-dimensional, intricately connected system. The response of Uranus to extremes of seasonal forcing due to its $98^{\circ}$ obliquity will provide vital tests of our general understanding of atmospheres and magnetospheres and how they couple through the ionosphere. The rings and satellites will provide stark contrasts to those of Jupiter and Saturn enabling the study of a ring system unlike any other in the Solar System. Such work has important implications for our understanding of gravitating discs and planet-disc interactions. Furthermore, Uranus is the most accessible Ice Giant at an average heliocentric distance of 19.2 AU. Table 2 
Table 2 The key scientific questions for UP and their relevance for ESA's Cosmic Vision 20152025 goals

\begin{tabular}{cll}
\hline Theme & Science question & Cosmic vision \\
\hline Uranus as an & What is the internal structure and composition of Uranus? & $1.1 / 1.2 / 1.3 / 2.2$ \\
Ice Giant & Why does Uranus emit very little heat? & $1.1 / 2.2$ \\
planet & What is the configuration \& origin of Uranus' magnetic field? & $1.3 / 2.1 / 2.2$ \\
& What is the rotation rate of Uranus? & $1.1 / 2.2$ \\
& How is Uranus' weather and composition influenced by season? & 2.2 \\
& What processes shape chemistry and cloud formation on an & 2.2 \\
& $\quad$ Ice Giant? & 2.2 \\
Uranus' & What is the composition of the uranian rings? & 2.2 \\
Ice Giant & How do dense rings behave dynamically? & 2.2 \\
planetary & How do Uranus' dusty rings work? & $2.2 / 2.3$ \\
system & How do the rings and inner satellites interact? & $1.1 / 2.2 / 2.3$ \\
& What is the nature and history of Uranus' moons? & $1.1 / 1.3 / 2.1$ \\
Uranus' & What is the overall configuration of the uranian magnetosphere? & \\
asymmetric & How do the magnetosphere \& ionosphere couple to the & $1.2 / 2.1$ \\
magneto- & solar wind? & 2.1 \\
sphere & How are auroral radio emissions generated at Ice Giants? & $1.3 / 2.1 / 2.3$ \\
Cruise phase & How does the outer heliosphere work? & \\
science & What can we learn from in situ observations of Centaurs? & \\
\hline
\end{tabular}

highlights the key science questions for UP and demonstrates each question's relevance for our exploration goals as expressed in ESA's Cosmic Vision 20152025 .

\section{Mission profile}

The next stage in the evolution of Ice Giant exploration requires an orbiter to expand on the flyby science carried out by Voyager 2. Uranus Pathfinder proposes to be the first spacecraft to enter orbit around an Ice Giant planet and undertake an orbital tour of an Ice Giant planetary system

\subsection{Launch, interplanetary transfer and orbit requirements}

Due to the M-class mission cost cap, launch vehicles for the M-class programme are restricted to Soyuz-Fregat, Rockot KM and Vega, of which only the former has the necessary performance to launch UP with a reasonable transfer duration. Soyuz-Fregat is highly reliable and proven technology and poses a low risk of failure. The baseline spacecraft design described in Section 5 is based on the Fregat ST fairing. There are a wide variety of launch opportunities for UP which are entirely compatible with the 2020-2022 launch window specified in the ESA M3 call.

Interplanetary transfers have been studied in detail. The UP interplanetary transfer utilises a sequence of gravity assists as is usual for deep space missions and many routes were identified. These included a variety of Venus, Earth, and Saturn gravity assists for example VVE (Venus-Venus-Earth), VEE, 
EVVE, VEES (Venus-Earth-Earth-Saturn) or VVEES with a variety of Earth resonance options. Delta-V requirements for a mission to Uranus are not significantly larger than for a mission to Saturn. Mars usually extends the transfer duration and Jupiter will not be in a favourable position over the M3 launch window. UP does not depend critically on any particular solution except for the demands that sufficient injected mass is available for the nominal scientific payload. Chemical propulsion has been assumed for these studies but solar electric propulsion is expected to yield improvements to the transfer time, available Uranus orbits, or available payload mass. All studied transfers assume a launch from Kourou.

Soyuz-Fregat is restricted to a small range of escape declinations it can efficiently access. In some cases an assumption was made that UP would inject into an equatorial geostationary transfer orbit (GTO). Escape is then achieved by the use of a propulsion module to achieve the required $V_{\text {inf }}$ and declination. This propulsion module separates from the remaining spacecraft before further deep space manoeuvres. A generic loss of $\Delta v$ to cover finite thrust and plane changing has been included in these escape sequences. The injection mass vs. $\mathrm{C}_{3}$ (characteristic energy) is consistent with ESOC analyses for Mars NEXT and Marco Polo missions.

Table 3 indicates several selections of interplanetary transfers. The duration of the interplanetary transfer is typically 15 years with a launch in 2021 and provides a spacecraft mass of $>\sim 800 \mathrm{~kg}$. Figure 7 illustrates one of these solutions.

The orbits provided by the transfers described above are almost polar (similar to the NASA Juno spacecraft at Jupiter) with a periapsis less than $2 \mathrm{R}_{\mathrm{U}}$, apoapsis between 123 and $391 \mathrm{R}_{\mathrm{U}}$, and periods between 60 and 313 days. These orbits are quite adequate for the science demands of UP although they

Table 3 Summary of the key characteristics of three selected interplanetary transfers for Uranus Pathfinder

\begin{tabular}{|c|c|c|c|}
\hline Launch & 2021 & 2021 & 2021 \\
\hline $\begin{array}{l}\text { Uranus Orbit Insertion } \\
\text { (UOI) }\end{array}$ & 2037 & 2036 & 2037 \\
\hline Transfer duration (years) & 15.5 & 15.0 & 15.8 \\
\hline Sequence & V-E-E-S & $\mathrm{V}-\mathrm{V}-\mathrm{E}-\mathrm{E}$ & $\mathrm{E}-\mathrm{V}-\mathrm{DV}-\mathrm{V}-\mathrm{E}$ \\
\hline \multirow[t]{3}{*}{ Transfer margins } & \multicolumn{2}{|c|}{$5 \%$ margin of delta- $\mathrm{V}$} & $5 \%$ fuel margin \\
\hline & \multicolumn{2}{|c|}{$100 \mathrm{~m} / \mathrm{s}$ delta- $\mathrm{V}$ for navigation } & $100 \mathrm{~m} / \mathrm{s}$ delta-V for launch \\
\hline & \multicolumn{2}{|c|}{$\begin{array}{l}\text { Loss factor of } 20 \% \text { applied } \\
\text { to capture delta-V. }\end{array}$} & $\begin{array}{l}\text { Dispersion error and navigation. } \\
5 \% \text { gravity loss. }\end{array}$ \\
\hline \multicolumn{4}{|l|}{ Orbit } \\
\hline Periapsis & \multicolumn{2}{|c|}{$1.8 \mathrm{R}_{\mathrm{U}}(45,000 \mathrm{~km})$} & $1.1 \mathrm{R}_{\mathrm{U}}(28,100 \mathrm{~km})$ \\
\hline Apoapsis & \multicolumn{2}{|c|}{$391 \mathrm{R}_{\mathrm{U}}\left(10^{7} \mathrm{~km}\right)$} & $123 \mathrm{R}_{\mathrm{U}}\left(3.1 \times 10^{6} \mathrm{~km}\right)$ \\
\hline Period & \multicolumn{2}{|c|}{313 days } & 60 days \\
\hline \multirow[t]{2}{*}{ Remarks } & \multicolumn{2}{|c|}{$\begin{array}{l}\text { Stays outside main rings during } \\
\text { ring plane crossing }\end{array}$} & $\begin{array}{l}\text { Inside } \mu \text { ring during ring plane } \\
\text { crossing. }\end{array}$ \\
\hline & \multicolumn{2}{|c|}{$\begin{array}{l}\text { Assumes launch to GTO with } \\
\text { additional propulsion stage for } \\
\text { escape (similar to Marco Polo) }\end{array}$} & $\begin{array}{l}\text { Direct escape-consistent with } \\
\text { Mars Express }\end{array}$ \\
\hline
\end{tabular}




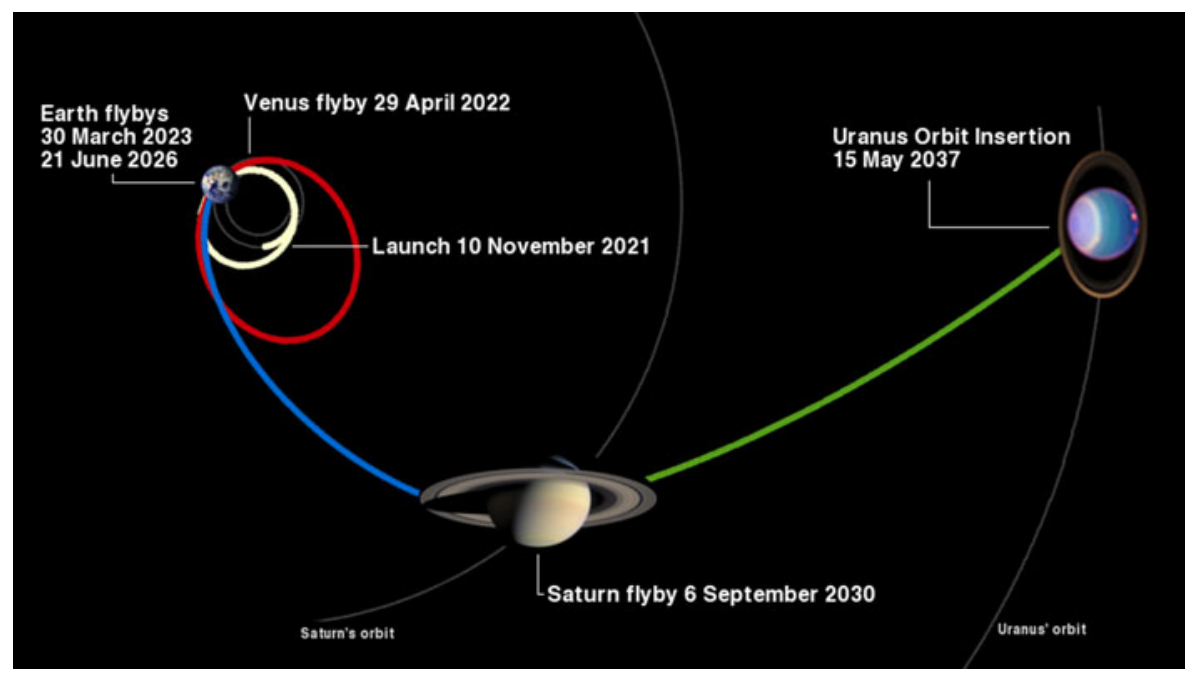

Fig. 7 Example trajectory for UP

complicate the development of an orbital tour for the uranian system. The details of such a satellite tour were not studied as part of the development of the UP concept but are of particular importance for Theme Two of the science case. The study of such a tour is a requirement for the assessment phase. Studies for the NASA Planetary Decadal Survey [34] have shown that such a tour is possible with a near-polar orbiting spacecraft. Close flybys of at least one of the major moons (preferably Titania or Oberon due to the possible presence of internal oceans) would represent an opportunity for significant advances in studying the origin and evolution of the natural satellites of Ice Giants.

The ring system of Uranus is poorly understood and presents a significant hazard uncertainty inside $52,000 \mathrm{~km}\left(2.06 \mathrm{R}_{\mathrm{U}}\right)$. In Table 3 we demonstrate an interplanetary transfer which has a periapsis at 28,100 $\mathrm{km}$ (but a ring plane crossing at $36,700 \mathrm{~km}$ inside the $\zeta$ ring). Such an option would be suitable for UP if more information on the ring system becomes available during the study phase. To improve our knowledge of Uranus' gravity field requires a periapsis inside $1.5 \mathrm{R}_{\mathrm{U}}$ where the spacecraft can be tracked outside of eclipseinside of $1.1 \mathrm{R}_{\mathrm{U}}$ there is sufficient drag from the atmosphere to degrade the gravity measurements. In principle the periapsis for two of the solutions in Table 3 could be reduced later in the mission thus permitting a more expanded programme of gravity science. This might be achieved using moon flybys.

The relatively low telemetry rates at Uranus' heliocentric distance require an orbital period sufficiently long to allow downlink of science data taken near periapsis. The orbits provided by the interplanetary transfer options in Table 3 span a range of reasonable options to satisfy these demands. Longer orbits also restrict the amount of data that can be taken since the power available 
Table 4 Telemetry rates and data volumes per downlink for a variety of ground stations and bands

\begin{tabular}{lllc}
\hline $\begin{array}{l}\text { Ground station } \\
\text { and band }\end{array}$ & $\begin{array}{l}\text { Figure of } \\
\text { merit }(\mathrm{dB})\end{array}$ & $\begin{array}{l}\text { Telemetry } \\
\text { rate }(\mathrm{kbit} / \mathrm{s})\end{array}$ & $\begin{array}{l}\text { Volume per } 8 \mathrm{~h} \\
\text { down-link }(\mathrm{Mbit})\end{array}$ \\
\hline New Norcia $(\mathrm{X})$ & 49.5 & 2.0 & 56 \\
Cebreros $(\mathrm{X})$ & 50.8 & 2.7 & 75 \\
Cebreros $(\mathrm{Ka})$ & 55.7 & 8.3 & 230 \\
\hline
\end{tabular}

from radioactive power sources will diminish over time, limiting the number of orbits that can be executed. During the assessment phase a trade study will be conducted to estimate the amount of science data obtained during periapsis as a function of the orbital period.

\subsection{Ground segment and mission operations concept}

Ground activities during the UP cruise phase must be minimised due to the long interplanetary transfer, with launch and early operations managed at low cost within ESOC following the model of Rosetta. ESA ground station usage will be limited to tracking and cruise data downlink every few weeks similar to New Horizons. The science operations centre (UPSOC) will be established during the six months prior to Uranus orbit insertion (UOI) to support important upstream observations before orbit insertion.

Telemetry, tracking and control for UP is based around X- and Ka-band communications to ESA ground stations. Table 4 shows estimates of telemetry rates and data volumes for UP. These estimates assume a $3.5 \mathrm{~m}$ high gain antenna (HGA) with $30 \mathrm{~W}$ power input and $50 \%$ travelling wave tube antenna efficiency ( $15 \mathrm{~W}$ transmitted power). We have calculated the telemetry rates and data volumes possible from two ESA ground stations in both $\mathrm{X}$ and $\mathrm{Ka}$ band. The table also shows the figures of merit (antenna gain/noise ratio) used for each station. In each case these telemetry rates have been subjected to a $20 \%$ margin. We obtain data volumes of between 56 and 230 Mbit per $8 \mathrm{~h}$ downlink. These (X-band) values are consistent with calculations for Laplace/EJSM/JGO scaled for Earth-Uranus distance and transmitter power. For UP we have conservatively baselined 75 Mbit per downlink over Ka band. This data volume is sufficient to meet the science goals set out in Section 2.

Figure 8 illustrates the ground segment for UP. The spacecraft will be managed by the mission operations centre (UPMOC) and will utilise existing ESA technologies for efficient mission management (e.g., SCOS2000). The science operations centre (UPSOC) will have responsibility for archiving, provision of quicklook data, and for providing the interface between the instrument teams and UPMOC. Observing plans will be developed by the instrument teams and UPSOC and passed to UPMOC for uplink to UP. Observing plans for each periapsis pass will be developed near apoapsis and uplinked on the inbound leg of each orbit. Mission operations during cruise will be minimised to reduce costs, with UP in a spin-stabilised survey mode monitoring the solar wind. 


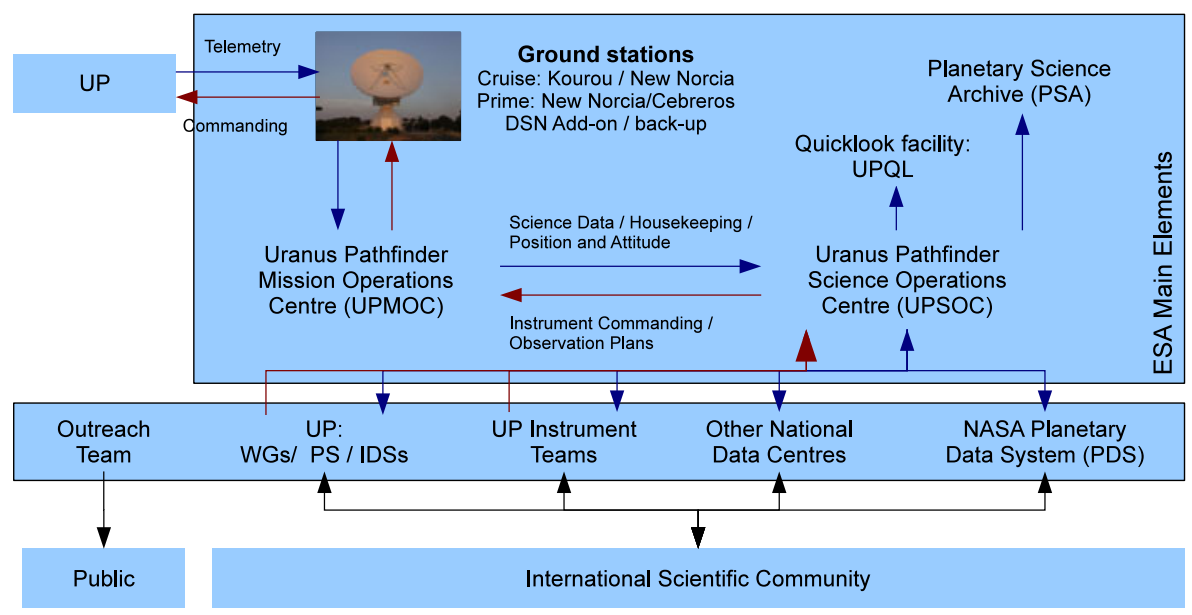

Fig. 8 Ground segment for UP

Science operations will be managed from an operations centre located at the European Space Operations Centre (ESOC). A system of project scientists, principal investigators, co-investigators, interdisciplinary scientists and working groups will be set up to exploit the huge science return from UP. The UP ground segment emphasises the significant interaction between the UP project and the wider scientific community, including specific community groups such as Europlanet. The data handling pipeline for UP follows the familiar and well-established pipeline for existing ESA missions (e.g., Mars Express, Venus Express, Rosetta, Cluster) and does not require additional development costs. Data will be stored on solid state recorders (SSR) on the spacecraft for regular downlink and will be processed by UPMOC and provided to UPSOC who will generate level 0 and quicklook data products, the former of which will be archived in ESA's Planetary Science Archive (PSA) and NASA's Planetary Data System (PDS). The quicklook data products will be served by a "quick look" service UPQL similar to the successful CSDS service implemented for the ESA Cluster mission. This will provide quick look access to raw imaging and time-series data to facilitate efficiently achieving the UP science goals. Level 0 data will be further calibrated and reduced by instrument teams who will provide higher level data products for archiving within PSA, PDS and other national data centres as appropriate. These higher level products will be provided a year after their receipt on the ground. A Data Archive Working Group and Archive Scientist will oversee this process. The data rights policy for UP is in compliance with established ESA rules concerning information and data rights and release policy. Instrument teams will have a proprietary six month period in which they can exploit their datasets after which the data will be placed in the public domain in PSA and PDS. 


\subsection{Support from ground-based observations}

Since Voyager 2 visited Uranus, scientists have relied on remote sensing observations from the ultraviolet through the microwave to constrain models of Uranus' atmosphere, rings and satellite system. These observations have been acquired by space-based observatories (Hubble, Spitzer, Herschel, ISO, etc.) and ground-based facilities (Keck, Gemini, VLT, IRTF and the VLA). In some cases these provide crucial information that could not be obtained from any reasonable Uranus orbiter (such as high spectral resolution). In other cases, they provide a long temporal baseline of contextual imagery to show how the uranian system evolves with time between spacecraft encounters.

Following well-established programmes of ground-based support for Galileo, Cassini, New Horizons and Juno, the UP consortium will apply for a sequence of regular observations from a range of observatories in the years preceding UOI. Observatories in the 8-10 m class (e.g., ESO/VLT, Subaru and Keck) could all contribute to the growing database of observations of Uranus. We also envisage enlisting the capabilities of the E-ELT (European Extremely Large Telescope), the ALMA sub-millimeter array and the TMT (Thirty Metre Telescope), as and when they can be tested for their sensitivity to Uranus. These observations will provide important contextual information for the UP mission and will extend UP's exploration beyond the nominal mission lifetime as ground-based observers follow up on the key discoveries of the ESA UP mission.

UP will also operate in synergy with other missions which may be flying in the 2036 timeframe, including the successors to the visible and infrared spacebased observatories of the coming decade (e.g., JWST, WFIRST), proposed US missions to the outer Solar System (e.g., Argo to Neptune/Triton, see [29] and [54]), and missions in the inner heliosphere.

\section{Proposed model scientific payload}

The UP model scientific payload incorporates a focused set of high TRL (technology readiness level) scientific instruments with heritage from recent missions (e.g., Cassini, Rosetta, Mars Express, Dawn, New Horizons) and future missions (e.g., Juno, Laplace/EJSM). To aid in managing the demands of a resource-limited spacecraft such as UP the scientific payloads will be combined following the model set by Rosetta. Careful placement of scientific instruments will also aid in making the most use of particular spacecraft attitudes-for example we envisage that all the optical remote sensing (ORS) instruments will be placed on the same side of the spacecraft and approximately boresighted similar to New Horizons and the Cassini orbiter. Table 5 documents the scientific payload for UP and shows the rich European flight heritage of this payload and its high TRL. The requirements of these instruments for 
Table 5 Model scientific payload for UP with TRL and heritage

\begin{tabular}{|c|c|c|}
\hline Instrument & TRL & Heritage \\
\hline Magnetometer (MAG) & 9 & $\begin{array}{l}\text { Cassini/MAG, } \\
\text { Double Star/MAG } \\
\text { Rosetta/RPC } \\
\text { Solar Orbiter }\end{array}$ \\
\hline Plasma and Particle Science (PPS) & $8 / 9$ & $\begin{array}{l}\text { Rosetta/RPC-IES } \\
\text { Cassini/CAPS-ELS } \\
\text { New Horizons/PEPPSI } \\
\text { THEMIS/SST }\end{array}$ \\
\hline Radio and Plasma Wave Experiment (RPW) & $8 / 9$ & $\begin{array}{l}\text { Cassini/RPWS, } \\
\text { STEREO/Waves, } \\
\text { RBSP, } \\
\text { BepiColombo/MMO/PWI }\end{array}$ \\
\hline Microwave Radiometer (MWR) & $7 / 8$ & Juno/MWR \\
\hline Thermal Infrared Bolometer (UTIRM) & 5 & $\begin{array}{l}\text { LRO/Diviner } \\
\text { BepiColombo (detectors) }\end{array}$ \\
\hline $\begin{array}{l}\text { Visual and Near-Infrared Mapping Spectrometer } \\
\text { (NIR/MSIC) }\end{array}$ & $>5$ & $\begin{array}{l}\text { New Horizons/RALPH } \\
\text { Mars Express/OMEGA } \\
\text { Juno/JIRAM Rosetta/VIRTIS } \\
\text { Dawn/VIR } \\
\text { Cassini/VIMS }\end{array}$ \\
\hline Ultraviolet Imaging Spectrometer (UVIS) & $>5$ & $\begin{array}{l}\text { BepiColombo/PHEBUS } \\
\text { Mars Express/SPICAM-UV } \\
\text { Venus Express/SPICAV-UV } \\
\text { Cassini/UVIS }\end{array}$ \\
\hline Narrow Angle Camera (NAC) & $>5$ & $\begin{array}{l}\text { EJSM-JGO/HRC } \\
\text { Mars Express/SRC } \\
\text { New Horizons/LORRI }\end{array}$ \\
\hline Radio Science Experiment (RSE) & 9 & $\begin{array}{l}\text { Venus Express/VeRa } \\
\text { Rosetta/RSI }\end{array}$ \\
\hline
\end{tabular}

A TRL of 5 indicates that the technology has been tested in a simulated environment, a TRL of 7 indicates the availability of a prototype that is close to the planned operational system, and a TRL of 9 indicates that the system in its final form has been used under actual mission conditions

meeting the scientific goals (Table 2) of UP are given in the traceability matrix in Table 6 . The total mass for these instruments, including appropriate design maturity margins ranging between $5 \%$ and $30 \%$, is $62.6 \mathrm{~kg}$ and they draw $88.1 \mathrm{~W}$ when fully operating.

\section{Spacecraft key issues and technological developments}

UP is compatible with existing mission platforms such as Rosetta and Mars/Venus Express [18, 24] and will be built using this existing heritage. The critical issues that drive the design of the spacecraft and mission are a) spacecraft mass, b) electrical power source, c) thermal control, d) expected data volumes and bandwidth, and e) minimising costs during the cruise phase. In this section we address these critical issues and some spacecraft design issues. 


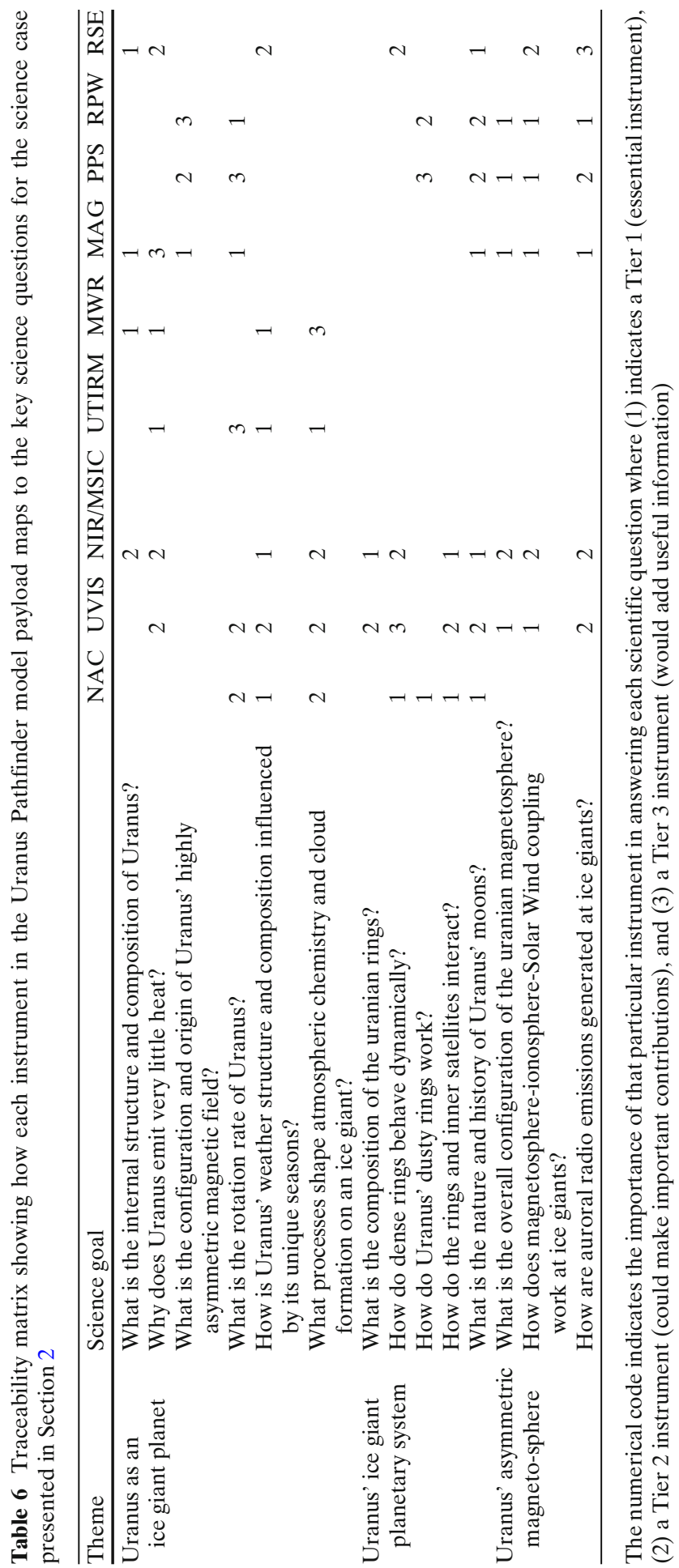




\subsection{Electrical power}

Estimating that $200 \mathrm{~W}$ of electrical power is required to operate the spacecraft platform and scientific payload we can estimate the area of solar panels required to operate UP as a solar powered mission. The power from the solar panels can be written as $\mathrm{P}=\varepsilon \mathrm{A}\left(\mathrm{L} / 4 \pi \mathrm{r}^{2}\right)$ where $\mathrm{L}$ is the luminosity of the Sun, $\mathrm{r}$ is the distance from the Sun to the Earth, $\varepsilon$ is the efficiency of the solar panels, and $\mathrm{A}$ is the area of the solar panels. Using the solar panels on Rosetta as a baseline [26] where $\mathrm{A}=64 \mathrm{~m}^{2}, \mathrm{r}=5.25 \mathrm{AU}$ and $\mathrm{P}=395 \mathrm{~W}$ we can write $\mathrm{P}_{\mathrm{UP}} / \mathrm{P}_{\text {Rosetta }}=(5.25 / 19.2)^{2} \mathrm{~A}_{\mathrm{UP}} / \mathrm{A}_{\text {Rosetta. }}$. Hence, following this scaling the UP solar panels need to be at least $440 \mathrm{~m}^{2}$ and in practice much higher due to the fall-off in solar panel efficiency at low intensities and low temperatures (LILT). This is not feasible within the M-class programme due to launch mass, low TRL for LILT solar arrays, and operational complexity. Hence, UP requires electrical power from radioactive power sources (RPS). This is the key technological development for UP and such technology is already in development through ESA contracts. The development of a European RPS system is driven by a) the costs of fuel production and the management of associated safety aspects, b) the requirement that these devices be at TRL 5-6 (including launch safety) by 2015, c) thermal and physical accommodation on a spacecraft, and d) operation for more than 15 years. These specifications make them viable candidates for UP.

In terms of radioisotopes, ${ }^{238} \mathrm{Pu}$ and ${ }^{241} \mathrm{Am}$ are the best candidates, although the decay heat per unit mass of ${ }^{241} \mathrm{Am}\left(0.11 \mathrm{~W} \mathrm{~kg}^{-1}\right)$ is $1 / 5$ that of ${ }^{238} \mathrm{Pu}\left(0.57 \mathrm{~W} \mathrm{~kg}^{-1}\right)$. This difference in efficiency must be managed at a system level which implies that a Stirling-type converter must be used for a ${ }^{241}$ Am-based device. The baseline RPS devices are based around ${ }^{241} \mathrm{Am}$ (in the form of $\mathrm{Am}_{2} \mathrm{O}_{3}$ ), which is obtained from processed spent fuel rods from nuclear reactors. The ${ }^{241} \mathrm{Am}$ is the decay product of ${ }^{241} \mathrm{Pu}$ (with a half life of 14.4 years) which is present in the form of $\mathrm{PuO}_{2}$ in spent fuel and so pure ${ }^{241} \mathrm{Am}$ is directly obtained and does not require complex processes to separate ${ }^{243} \mathrm{Am}$ and ${ }^{241} \mathrm{Am}$ directly from the spent fuel rods. This processed spent fuel has been in storage in France and the United Kingdom for 20 years and thus a supply for pure ${ }^{241} \mathrm{Am}$ is readily available within Europe and will not be a barrier to the use of an ESA RPS on UP (see the following press release for more information http://www.nnl.co.uk/news/ nnl-wins-1-million-space-batteries-contract.html Accessed 26 July 2011).

Should this programme fail to produce a viable RPS unit in time for the M3 programme our mitigation strategy is to use a NASA-provided MultiMission Radioisotope Thermoelectric Generator (MMRTG) or Advanced Stirling Radioisotope Generator (ASRG) device to power the spacecraft and scientific payload. Our power and mass budgets allow for this eventuality, and the switch to this alternative power source does not present a mission-critical issue, nor does it affect the ability of the mission to carry out its scientific programme. As noted above, the specific power of an ${ }^{241} \mathrm{Am}$-based device is less than that of a ${ }^{238} \mathrm{Pu}$-based device because the specific thermal power of 
${ }^{238} \mathrm{Pu}$ is five times that of ${ }^{241} \mathrm{Am}$. Also, ${ }^{241} \mathrm{Am}$ is a more prodigious neutron and gamma ray emitter than ${ }^{238} \mathrm{Pu}$ thus requiring more shielding mass. Hence, the use of a ${ }^{241} \mathrm{Am}$ device represents a "worst case" scenario in terms of specific electrical power; switching to an alternative MMRTG or ASRG device represents a gain in platform/payload mass and available electrical power.

\subsection{Thermal control}

Thermal control is an important driver of every mission and UP is no exception; for UP this is challenging due to extreme differences in thermal environment between Venus and Uranus and the continuous supply of thermal energy from RPS units. Such thermal control issues can be adequately managed by modifying existing designs from Rosetta and Mars/Venus Express. Established combinations of heaters, radiators and louvers will enable these thermal issues to be addressed. We have estimated that $\sim 45 \mathrm{~W}$ will be required to maintain an internal spacecraft temperature of $-10^{\circ} \mathrm{C}$ against losses to space. This estimate is based on a spacecraft of similar size to Mars Express covered with multilayer insulation (MLI). We do not assume that this power can be derived from dissipation of heat from internal equipment and include $45 \mathrm{~W}$ in the power budget for electrical heaters (in addition to instrument heaters). Efficient mission operations will ease these demands. Shunt resistors to manage the power from the RPS units can be externally or internally mounted to help heat the spacecraft. Spot heating might be provided by radioactive heating units (RHU), potentially based on ${ }^{241} \mathrm{Am}$.

\subsection{Planetary protection}

Planetary projection requirements are less stringent at Uranus permitting the use of existing spacecraft bus designs (e.g., Rosetta, Mars Express). Uranus is listed as Class II for planetary protection purposes and so the study phase only requires mission analysis and design to minimise the risk of a collision between the orbiter and any sites of potential prebiotic interest, such as the moons Titania and Oberon.

\subsection{Radiation constraints}

Uranus has a fairly benign radiation environment (compared to Jupiter) and has radiation belts of roughly the same intensity as Saturn but which are less intense than at Earth. SPENVIS (SHIELDDOSE-2) was used to estimate a total mission radiation dose of $20 \mathrm{kRad}$ behind $4 \mathrm{~mm}$ of aluminium. Most of this dose comes from the cruise phase $(18 \mathrm{kRad})$ and was estimated from nearEarth interplanetary space. The radiation dose per orbit of Uranus $(0.2 \mathrm{kRad})$ was estimated from terrestrial radiation models with the UP orbits scaled down by the relative planetary sizes. This gives a dose of $2 \mathrm{kRad}$ for the prime mission of ten orbits. 


\subsection{Attitude and orbit control}

Attitude and orbit control (AOCS) for UP will be achieved by a combination of thrusters and reaction wheels with solid heritage from Mars/Venus Express and Rosetta. During cruise phase the spacecraft will be spin stabilised to minimise deterioration of the reaction wheels and simplify operations. During the prime mission UP will be three-axis stabilised using a combination of reaction wheels and thrusters. Three-axis stabilisation is required for the relatively long integration times required by ORS instruments. The use of RPS units for electrical power gives UP a low inertia compared to a spacecraft using solar arrays thereby allowing UP to slew rapidly to view multiple targets.

\subsection{On-board data management}

On-board processing will be restricted due to mission mass and power constraints but each instrument, particularly those that operate in a survey mode such as the magnetometer, will have some intelligent processing capability able to retain interesting data at a higher cadence than nominal. The estimated data volumes total 4.1 Gbit per orbit. On-board storage of data on SSRs for downlink at a later date is common amongst deep space missions and UP will use solutions similar to Venus Express and Rosetta; UP will have 12 Gbit of on-board capacity in three 4-Gbit SSR modules, facilitating redundancy in case

Table 7 Power budget for Uranus Pathfinder including design maturity margins

\begin{tabular}{lccc}
\hline Subsystem & Downlink [W] & MAG/RPW/PPS [W] & ORS [W] \\
\hline Platform & 162 & 132 & 132 \\
Avionics & 24 & 24 & 24 \\
Communications & 36 & 6 & 6 \\
AOCS & 24 & 24 & 24 \\
Propulsion actuators & 12 & 12 & 12 \\
Thermal control & 54 & 54 & 54 \\
Power & 12 & 12 & 12 \\
Science payload & 21.8 & 36.6 & 56.4 \\
MAG & $2^{*}$ & 5.3 & 2 \\
PPS & $2^{*}$ & 7.7 & $2^{*}$ \\
RPW & $2^{*}$ & 7.8 & $2^{*}$ \\
MWR & $2^{*}$ & $2^{*}$ & $2^{*}$ \\
UTIRM & $2^{*}$ & $2^{*}$ & 5.2 \\
NIR/MSIC & $2^{*}$ & $2^{*}$ & 7.8 \\
UVIS & $2^{*}$ & $2^{*}$ & 14.4 \\
NAC & $2^{*}$ & $2^{*}$ & 15.2 \\
RSE & 5.8 & 5.8 & 5.8 \\
Total & 183.8 & 168.6 & 188.4 \\
Net & 8.2 & 23.4 & 3.6 \\
\hline
\end{tabular}

All powers are given in watts and include margins on the power source (providing $192 \mathrm{~W}$ maximum including margin) and on each individual instrument. Because the $192 \mathrm{~W}$ provided by the RPS units cannot operate all instruments simultaneously a number of operational scenarios are given and shows that the power supplied by the RPS units is sufficient to enable science operations 
of the failure of a module. UP can downlink 75 Mbit per day (Table 4). Over a 60 -day orbit, where downlinking only occurs on 56 days to account for periapsis science operations, 4.2 Gbit can be downlinked exceeding the demands of the scientific payload. The mission would still be viable if two of the three SSRs failed.

\subsection{System budgets}

Our estimate of the available power from two RPS units is 192 W including margins. During downlink manoeuvres we estimate that the platform draws $162 \mathrm{~W}$ whilst nominally drawing $132 \mathrm{~W}$. Clearly this requires significant observation planning and resource management since the full scientific payload draws $88 \mathrm{~W}$. Table 7 presents several examples of operational power management scenarios during different spacecraft operations/scientific campaigns.

The total dry mass for UP (including all margins) evaluated to $836.1 \mathrm{~kg}$ and meets the launch capability of Soyuz-Fregat with an $8 \%$ margin (see Table 8

Table 8 Mass budget for Uranus Pathfinder including design maturity margins

\begin{tabular}{lc}
\hline Subsystem & $\begin{array}{c}\text { Mass (including } \\
\text { margin) [kg] }\end{array}$ \\
\hline Scientific payload & 62.6 \\
Magnetometer (MAG) & 2.2 \\
Plasma and Particle Science (PPS) & 7.2 \\
Radio and Plasma Wave Experiment (RPW) & 6.3 \\
Microwave Radiometer (MWR) & 14.4 \\
Thermal Infrared Bolometer (UTIRM) & 1.3 \\
Visual and Near-Infrared Mapping Spectrometer (NIR/MSIC) & 11.6 \\
Ultraviolet Imaging Spectrometer (UVIS) & 6 \\
Narrow Angle Camera (NAC) & 12 \\
Radio Science Experiment (RSE) & 1.6 \\
Radioisotope power system & 192 \\
Structure (inc. harness and mechanisms) & 81.6 \\
Communications & 60 \\
Data handling & 24 \\
AOCS & 60 \\
AOCS fuel & 40 \\
Thermal control & 48 \\
Radiation shielding & 12 \\
Propulsion and associated structure & 116.2 \\
Nominal dry spacecraft mass & 696.8 \\
System level margin (20\%) & 139.4 \\
Total dry mass & 836.1 \\
Propellant mass & 690.1 \\
Total wet mass & 1526.3 \\
Escape stage mass (inc. margin) & 218 \\
Escape stage propellant & 1080.4 \\
Total launch mass & 2824.7 \\
Launch vehicle performance & 3080 \\
Excess launch capability & $255.8(8 \%)$ \\
\hline & \\
M & \\
\hline &
\end{tabular}

Minor differences between the quoted total masses and those obtained from summing the masses of the components are due to rounding values from the mass budget spreadsheet 
for the mass budget for UP). The overall system configuration was designed around a Mars Express-type platform and so it is not entirely unexpected that the total dry mass is very similar to that for Mars Express. This clearly shows that important and distant Solar System targets can be reached by a SoyuzFregat launch vehicle.

\section{Communications and outreach}

A European mission to a mysterious and distant world like Uranus provides a unique public engagement opportunity. Pictures of distant bodies in the Solar System capture the public's imagination and attract school children and higher-level students to physics and astronomy. Planetary research also continues to grab headlines in the press, both in traditional print and new media. Uranus' moons are named after literary characters from the works of William Shakespeare and Alexander Pope, providing a particularly exciting opportunity to engage with a wider community than any previous mission by exploiting this link to the arts. We envisage a range of activities, particularly in schools and linked to national educational curricula.

Europe has extensive expertise and experience in delivering an outreach programme centred on giant planets through the ESA-NASA CassiniHuygens mission. The UK in particular has had many successes in engaging the public in Cassini-Huygens through programmes organised through the Royal Observatory, Greenwich and the Royal Astronomical Society, and also recently in a variety of activities related to the ESA Herschel mission. Outreach in the amateur astronomy community would also enable interesting and potentially valuable "citizen science projects". The outreach team will also utilise links with national public engagement stakeholders (e.g., Germany Physical Society, Royal Society, European Space Education and Research Office).

The long duration of the UP mission provides an excellent public engagement opportunity in which school children "Pathfinder kids" can follow the mission developments as they proceed through their classes learning evermore details about planetary exploration and the processes occurring therein. Special public engagement campaigns centred around key mission milestones such as the gravity assists and UOI will maintain public interest and awareness. This also provides a perfect example for showing the public the length of space missions necessitated by the enormous scale of the solar system, but also the resulting ambitious goals that can be achieved.

\section{Conclusions}

Uranus is an enigmatic world of extremes, a key component of our Solar System that the Cosmic Vision should seek to explore and explain if we hope to understand the origins, evolution and fundamental physicochemical 
processes, both in our planetary system and in those around other stars. Exploring the physicochemical processes at work within our Solar System will provide insights into its formation and evolution, helping to answer scientific questions of the highest importance, including some of the main objectives of ESA's Cosmic Vision 2015-2025. A mission to the Uranus system directly addresses important aspects of two of the Cosmic Vision themes: "What are the conditions for Planet Formation and the Emergence of Life?" and "How Does the Solar System Work?" Furthermore, in addressing the origins and evolution of Uranus-mass objects we directly address topics that are important for current and future exoplanet research. The use of a comprehensive but focused suite of advanced scientific instrumentation on a robust ESA orbiter, with significant flight heritage from Rosetta, Mars/Venus Express and BepiColombo, will provide significant potential for new discoveries and solutions to unresolved questions on the frontier of the outer Solar System.

UP can be implemented effectively using existing spacecraft platforms such as Mars Express/Rosetta but can also significantly drive technology developments such as European capability in radioisotope power and heat sources. Similar to any space mission, UP obviously benefits from international collaboration. In the case of UP this would enable a larger mission, shorter interplanetary transfer, the possibility for an atmospheric descent probe, and the leverage of international expertise which is naturally spread across the globe. The UP Consortium contains the complete body of expertise for successful exploitation of an Ice Giant orbiter mission. The UP mission concept reveals how much can be achieved within the ESA "medium-class" mission cost cap and demonstrates the heights to which ESA's Cosmic Vision can and should reach.

Although UP has not been selected for the assessment phase for the M3 programme a Uranus mission has been highly rated by the 2011 NRC Planetary Decadal Survey 2013-2023 with a Uranus "flagship" class mission rated third in priority. Future European opportunities will be exploited should a NASAled Uranus mission not be selected. The UP mission concept and science case demonstrates the need to explore the outer Solar System and the technical challenges which that entails. Technological advances in the fields of low-mass instrumentation, solar power, radioisotope power sources, and ion propulsion will enable such missions to be carried out whilst lowering risk and cost.

Acknowledgements CSA was supported by a Science and Technology Facilities Council Postdoctoral Fellowship. LNF was supported by a Glasstone Fellowship at the University of Oxford. We wish to thank EADS Astrium and Systems Engineering and Assessment Ltd. for their assistance with this study.

\section{Appendix A}

The 165 individuals (109 in Europe, in 67 institutes in 13 countries) listed below support the UP mission. 


\section{Argentina}

Instituto de Astronomía y Física del Espacio

Cèsar Bertucci

\section{Belgium}

Royal Observatory of Belgium

Ozgur Karatekin

Université de Liège

Aikaterini Radioti, Bertrand Bonfond, Denis Grodent, Jacques Gustin,

Jean-Claude Gérard

University of Namur

Benoît Noyelles

\section{Czech Republic}

Institute of Atmospheric Physics, Prague

Benjamin Grison, Jan Soucek, Ondrej Santolik, Vratislav Krupar

\section{France}

Centre d'Etude Spatiale des Rayonnements

Nicolas André

CNRS-UJF, Laboratoire de Planétologie de Grenoble, Bâtiment $D$ de physique, Grenoble

Mathieu Barthélémy

IAS, Université Paris Sud

Yves Langevin

\section{IMCCE}

Valery Lainey, Jacques Laskar, Nicolas Rambaux

Laboratoire AIM, Université Paris Diderot/CEA/CNRS

Sebastien Charnoz

\section{LATMOS}

Francois Leblanc, Eric Quémerais

LESIA, L'Observatoire de Paris

Baptiste Cecconi, Carine Briand, Daniel Gautier, Laurent Lamy, Olga Alexandrova, Philippe Zarka, Pierre Henri, Regis Courtin, Renée Prangé, Sandrine Vinatier

LPCEE, Orléans

Aurélie Marchaudon

LPG, CNRS-Université de Nantes

Gabriel Tobie

LPP, Ecole Polytechnique

Patrick Canu

Observatoire de Besançon

Jean-Marc Petit, Olivier Mousis

ONERA 
Christophe Bruno

Université de Versailles

Ronan Modolo

\section{Germany}

$D L R$

Frank Sohl

Freie Universität Berlin

Gerhard Neukum, Stephan van Gasselt

Max Planck Institute for Solar System Research

Ulrich Christensen, Norbert Krupp, Elias Roussos

Technical University Braunschweig

Karl-Heinz Glassmeier

Universität der Bundeswehr München

Thomas Andert, Bernd Häusler

University of Cologne

Fritz M Neubauer, Martin Pätzold, Joachim Saur, Silvia Tellmann

Universität Rostock

Nadine Nettelmann

\section{Greece}

Academy of Athens

Nick Sergis

\section{Hungary}

KFKI Research Institute for Particle \& Nucl. Physics

Geza Erdös, Karoly Szego, Sándor Szalai

\section{Israel}

Tel Aviv University

Aharon Eviatar

\section{Japan}

$J A X A$

Sarah Badman, Satoshi Kasahara

\section{Spain}

Centro de Astrobiologia, Madrid

Javier Martin-Torres

ESA ESAC

Stefan Remus

University of the Basque Country

Ricardo Huesco, Santiago Perez-Hoyos, Agustín Sánchez-Lavega 


\section{Sweden}

Swedish Institute of Space Physics, Kiruna

Stas Barabash, Yasir Soobiah

Swedish Institute of Space Physics, Uppsala

Jan-Erik Wahlund

\section{United Kingdom}

Aberystwyth University

Mario Bisi, Andy Breen, Tony Cook

Armagh Observatory

Tolis Christou

Imperial College London

Leah-Nani S. Alconcel, Michele K. Dougherty, Marina Galand, Caitríona

M. Jackman, Daniel Went, Ingo Müller-Wodarg

Open University

Dave Rothery

Queen Mary University of London

Carl D. Murray, Craig B. Agnor

University College London

Nicholas Achilleos, Chris Arridge, Andrew Coates, M. Entradas, Andrew

Fazakerley, Colin Forsyth, A. Dominic Fortes, Patrick Guio, Geraint H.

Jones, Sheila Kanani, Gethyn R Lewis, Steve Miller, Adam Masters,

Chris Owen, Alan Smith, Andrew P. Walsh

University of Bristol

Nick Teanby

University of Leicester

David Andrews, Emma Bunce, Stanley W H Cowley, Stephanie Kellett,

Henrik Melin, Steve Milan, Jon Nichols, Tom Stallard

University of Liverpool

Richard Holme

University of Oxford

Neil Bowles, Leigh Fletcher, Pat Irwin

University of Reading

Matt Owens

\section{United States of America}

Boston University

Supriya Chakrabarti, Luke Moore

Cornell University

Don Banfield, Matt Hedman, Matthew Tiscareno, Phil Nicholson

Georgia Tech

Carol Paty

Gordon College

Richard W. Schmude, Jr.

Johns Hopkins University-APL 
Pontus Brandt, Andrew Cheng, Chris Paranicas, Abigail M Rymer, H. Todd Smith, Elizabeth P Turtle

LPI, University of Arizona

Robert H Brown, Paul Schenk

NASA Goddard Space Flight Centre

Carrie M Anderson, Matt Burger, Glyn Collinson, John F Cooper, Brigette Hesman, Edward C Sittler

NASA Jet Propulsion Laboratory

Kevin Baines, A. Jim Friedson, Mark Hofstadter, Conor Nixon, Jim

Norwood, Glenn Orton, Robert T Pappalardo, Ed Smith

New Mexico State University

Reta Beebe, Nancy Chanover

Rice University

Tom Hill

SETI Institute

Mark Showalter

Southwest Research Institute, San Antonio

Scott Bolton, Mihir Desai, Dave McComas, Prachet Mokashi, Daniel Santos-Costa

Space Science Institute (Boulder)

Julianne Moses

University of California, Berkeley

Imke de Pater

University of California Los Angeles

Jerry Schubert, Ravit Helled, Chris Russell, Krishan Khurana, Margaret

Kivelson, Kunio Sayanagi

University of California Santa Cruz.

Jonathan Fortney

University of Colorado, Boulder

Sébastien Hess, Rob Wilson

University of Iowa

Jared Leisner, William Kurth, Patricia Schippers, Ulrich Taubenschuss

Washington University

Bill McKinnon

\section{References}

1. Ballester, G.E.: Magneotspheric interactions in the major planets. In: Wamsteker, W., Gonzalez Riestra, R. (eds.) Ultraviolet Astrophysics Beyond the IUE Final Archive, Proceedings of the Conference held in Sevilla, Spain, from 11-14 November 1997, ESA SP, vol. 413, p. 21. ESA Publications Division (1998)

2. Behannon, K.W., Lepping, R.P., Sittler Jr., E.C., Ness, N.F., Mauk, B.H.: The magnetotail of Uranus. J. Geophys. Res. 92(A13), 15354-15366 (1987). doi:10.1029/JA092iA13p15354

3. Belton, M.J.S., et al.: New Frontiers in the Solar System: An Integrated Exploration Strategy. Solar System Exploration Survey Space Studies Board, National Research Council. The National Academies Press, Washington, D.C.. (2003) ISBN: 0-309-50836-3 
4. Bergstralh, J.T., Miner, E.D., Matthews, M.S. (eds.): Uranus. University of Arizona Space Science Series. University of Arizona Press. (1991) ISBN: 978-0816512089

5. Boué, G., Laskar, J.: A collisionless scenario for Uranus tilting. Astrophys. J. 712, L44 (2010). doi:10.1088/2041-8205/712/1/L44

6. Capone, L.A., Whitten, R.C., Prasad, S.S., Dubach, J.: The ionospheres of Saturn, Uranus, and Neptune. Astrophys. J. 215, 977-983 (1977). doi:10.1086/155434

7. Chandler, M.O., Waite, J.H.: The ionosphere of Uranus-a myriad of possibilities. Geophys. Res. Lett. 13, 6-9 (1986). doi:10.1029/GL013i001p00006

8. Christensen, U.R., Tilgner, A.: Power requirement of the geodynamo from ohmic losses in numerical and laboratory dynamos. Nature 429(6988), 169-171 (2004). doi:10.1038/nature02508

9. Crida, A.: Minimum mass solar nebulae and planetary migration. Astrophys. J. 698, 606-614 (2009). doi:10.1088/0004-637X/698/1/606

10. Croft, S.K., Soderblom, L.A.: Geology of the Uranian satellites. In: Bergstrahl, J.T., Miner, E.D., Matthews, M.S. (eds.) Uranus, pp. 561-628. University of Arizona Press, Tucson (1991)

11. de Pater, I., Hammel, H.B., Gibbard, S.G., Showalter, M.R.: New dust belts of Uranus: one ring, two ring, red ring, blue ring. Science 312(5770), 92-94 (2006). doi:10.1126/science. 1125110

12. de Pater, I., Hammel, H.B., Showalter, M.R., van Dam, M.A.: The dark side of the rings of Uranus. Science 317(5846), 1888 (2007). doi:10.1126/science.1148103

13. Delsanti, A., Jewitt, D.: The solar system beyond the planets. In: Blondel, Ph., Mason, J. (eds.) Solar System Update, pp. 267-294. Springer, Germany (2006)

14. Desch, M.D., Connerney, J.E.P., Kaiser, M.L.: The rotation period of Uranus. Nature 322, 42-43 (1986)

15. Desch, M.D., Kaiser, M.L., Zarka, P., Lecacheux, A., Leblanc, Y., Aubier, M., Ortega-Molina, A.: Uranus as a radio source. In: Bergstrahl, J.T., Miner, E.D., Matthews, M.S. (eds.) Uranus. University of Arizona Press, Tucson (1991)

16. Duncan, M.J., Lissauer, J.J.: Orbital stability of the uranian satellite system. Icarus 125(1), 1-12 (1997). doi:10.1006/icar.1996.5568

17. Elliot, J.L., Nicholson, P.D.: The rings of Uranus. In: Brahic, A., Greenberg, R. (eds.) Planetary Rings. University of Arizona Press, Tucson (1984)

18. Ferri, P., Denis, M.: Utilising Rosetta commonality to reduce mission operations cost for Mars Express. Acta Astronaut. 52(2-6), 353-359 (2003). doi:10.1016/S0094-5765(02)00175-3

19. Fortney, J., Nettelmann, N.: The interior structure, composition, and evolution of giant planets. Space Sci. Rev. 152(1-4), 423-447 (2010). doi:10.1007/s11214-009-9582-x

20. Fortney, J.J., Ikoma, M., Nettelmann, N., Guillot, T., Marley, M.S.: Self-consistent model atmospheres and the cooling of the solar system's giant planets. Astrophys. J. 729, 32 (2011). doi:10.1088/0004-637X/729/1/32

21. Fountain, G.H., Kusnierkiewicz, D.Y., Hersman, C.B., Herder, T.S., Coughlin, T.B., Gibson, W.C., Clancy, D.A., DeBoy, C.C., Hill, T.A., Kinnison, J.D., Mehoke, D.S., Ottman, G.K., Rogers, G.D., Stern, S.A., Stratton, J.M., Vernon, S.R., Williams, S.P.: The New Horizons spacecraft. Space Sci. Rev. 140, 23-47 (2008). doi:10.1007/s11214-008-9374-8

22. French, et al.: Dynamics and structure of the uranian rings. In: Bergstrahl, J.T., Miner, E.D., Matthews, M.S. (eds.) Uranus, pp. 327-409. University of Arizona Press, Tucson (1991)

23. Geissler, P.E., Greenberg, R., Hoppa, G., et al.: Evidence for non-synchronous rotation of Europa. Nature 391, 368 (1998)

24. Gimenez, A., Lebreton, J.-P., Svedhem, H., Tauber, J.: Studies on the re-use of the Mars Express platform. ESA Bull. 109, 78-86 (2002)

25. Gladman, B., Quinn, D.D., Nicholson, P., Rand, R.: Synchronous locking of tidally evolving satellites. Icarus 122(1), 166-192 (1996). doi:10.1006/icar.1996.0117

26. Glassmeier, K.-H., Boehnhardt, H., Koschny, D., Kürt, E., Richter, I.: The Rosetta mission: flying towards the origin of the solar system. Space Sci. Rev. 128, 1-21 (2007). doi:10.1007/ s11214-006-9140-8

27. Guillot, T.: The interiors of giant planets: models and outstanding question. Ann. Rev. Earth Planet. Sci. 33, 493-530 (2005)

28. Hammel, H.B., Sromovsky, L.A., Fry, P.M., Rages, K., Showalter, M., de Pater, I., van Dam, M.A., Lebeau, R.P., Deng, X.: The dark spot in the atmosphere of Uranus in 2006: discovery, description, and dynamical simulations. Icarus 201(1), 257-271 (2008) 
29. Hansen, C.J., Aljabri, A.S., Banfield, D., Bierhaus, E.B., Brown, M., Colwell, J.E., Dougherty, M., Hendrix, A.R., Ingersoll, A., Khurana, K., Landau, D., McEwen, A., Paige, D.A., Paranicas, C., Satter, C.M., Schmidt, B., Showalter, M., Spilker, L.J., Spilker, T., Stansberry, J., Strange, N., Tiscareno, M.: Neptune science with Argo-a voyage through the outer solar system. White paper submitted to the 2013-2022 Planetary Decadal Survey. http://www8.nationalacademies.org/ssbsurvey/DetailFileDisplay.aspx?id=37\&parm_type=PSDS (2011). Accessed 20 July 2011

30. Helled, R., Anderson, J.D., Schubert, G.: Uranus and Neptune: shape and rotation. Icarus 210(1), 446-454 (2010). doi:10.1016/j.icarus.2010.06.037

31. Herbert, F.: Aurora and magnetic field of Uranus. J. Geophys. Res. 114, A11206 (2009). doi:10.1029/2009JA014394

32. Herbert, F., Sandel, B.R.: The uranian aurora and its relationship to the magnetosphere. J. Geophys. Res. 99(A3), 4143-4160 (1994)

33. Holme, R., Bloxham, J.: The magnetic fields of Uranus and Neptune: methods and models. J. Geophys. Res. 101(E1), 2177-2200 (1996). doi:10.1029/95JE03437

34. Hubbard, W.R., et al.: Ice giants decadal study. http://sites.nationalacademies.org/SSB/SSB_ 059331(2010). Retrieved 7 October 2010

35. Hussmann, H., Sohl, F., Spohn, T.: Subsurface oceans and deep interiors of medium-sized outer planet satellites and large trans-neptunian objects. Icarus 185(1), 258-273 (2006). doi:10.1016/j.icarus.2006.06.005

36. Jacobson, R.A., Campbell, J.K., Taylor, A.H., Synnott, S.P.: The masses of Uranus and its major satellites from Voyager tracking data and Earth-based uranian satellite data. Astron. J. 103(6), 2068-2078 (1992)

37. Lorenz, R.D., Stiles, B.W., Kirk, R.L. et al.: Titan's rotation reveals an internal ocean and changing zonal winds. Science 1649-1651 (2008)

38. Majeed, T., Waite, J.H., Bougher, S.W., Yelle, R.V., Gladstone, G.R., McConnell, J.C., Bhardwaj, A.: The ionospheres-thermospheres of the giant planets. Adv. Space Res. 33(2), 197-211 (2004). doi:10.1016/j.asr.2003.05.009

39. Melin, H., Stallard, T., Miller, S., Trafton, L.M., Encrenaz, Th, Geballe, T.R.: Seasonal variability in the ionosphere of Uranus. Astrophys. J. 729, 134 (2011). doi:10.1088/ 0004-637X/729/2/134

40. Murray, C.D., Thompson, R.P.: Orbits of shepherd satellites deduced from the structure of the rings of Uranus. Nature 348, 499-502 (1990). doi:10.1038/348499a0

41. Ness, N.F., Connerney, J.E.P., Lepping, R.P., Schulz, M., Voigt, G.-H.: The magnetic field and magnetospheric configuration of Uranus. In: Bergstrahl, J.T., Miner, E.D., Matthews, M.S. (eds.) Uranus, pp. 739-779. University of Arizona Press, Tucson (1991)

42. Peale, S.J., Phillips, R.J., Solomon, S.C., Smith, D.E., Zuber, M.T.: A procedure for determining the nature of Mercury's core. Meteorit. Planet. Sci. 37, 1269-1283 (2002)

43. Pearl, J.C., Conrath, B.J., Hanel, R.A., Pirraglia, J.A.: The albedo, effective temperature, and energy balance of Uranus, as determined from Voyager IRIS data. Icarus 84, 12-28 (1990). doi:10.1016/0019-1035(90)90155-3

44. Plescia, J.B.: Cratering history of the Uranian satellites-Umbriel, Titania, and Oberon. J. Geophys. Res. 92, 14918-14932 (1987). doi:10.1029/JA092iA13p14918

45. Saumon, D., Guillot, T.: Shock compression of deuterium and the interiors of Jupiter and Saturn. Astrophys. J. 609(2), 1170-1180 (2004). doi:10.1086/421257

46. Saur, J., Neubauer, F.M., Glassmeier, K.-H.: Induced magnetic fields in solar system bodies. Space Sci. Rev. 152, 391-421 (2010). doi:10.1007/s11214-009-9581-y

47. Sayanagi, K.M., Showman, A.P., Dowling, T.E.: The emergence of multiple robust zonal jets from freely evolving, three-dimensional stratified geostrophic turbulence with applications to Jupiter. J. Atmos. Sci. 65(12), 3947 (2008). doi:10.1175/2008JAS2558.1

48. Seidelmann, P.K., Archinal, B.A., A'hearn, M.F., Conrad, A., Consolmagno, G.J., Hestroffer, D., Hilton, J.L., Krasinsky, G.A., Neumann, G., Obsert, J., Stooke, P., Tedesco, E.F., Tholen, D.J., Thomas, P.C., Williams, I.P.: Report of the IAU/IAG working group on cartographic coordinates and rotational elements: 2006. Celest. Mech. Dyn. Astr. 98, 155-180 (2007). doi:10.1007/s10569-007-9072-y

49. Selesnick, R.S., Richardson, J.D.: Plasmasphere formation in arbitrarily oriented magnetospheres. Geophys. Res. Lett. 13, 624-627 (1986). doi:10.1029/GL013i007p00624 
50. Showalter, M., Lissauer, J.: The second ring-moon system of Uranus: discovery and dynamics. Science 311(5763), 973-977 (2006). doi:10.1126/science.1122882

51. Showalter, M.R., Lissauer, J.J., French, R.G., Hamilton, D.P., Nicholson, P.D., de Pater, I., Dason, R.: HST observations of the uranian outer ring-moon system. Bull.-Am. Astron. Soc. 40, $431(2008)$

52. Showman, A.P.: Numerical simulations of forced shallow-water turbulence: effects of moist convection on the large-scale circulation of Jupiter and Saturn. J. Atmos. Sci. 64, 3132-3157 (2007)

53. Sittler Jr., E.C., Ogilvie, K.W., Selesnick, R.: Survey of electrons in the Uranian magnetosphere: Voyager 2 observations. J. Geophys. Res. 92, 15263 (1987)

54. Squyres, S., et al.: Vision and Voyages for Planetary Science in the Decade 2013-2022. Committee on the Planetary Science Decadal Survey; National Research Council. Published by The National Academies Press, Washington, D.C.. ISBN: 0-309-20955-2. Pre-publication version (2011)

55. Stanley, S., Bloxham, J.: Convective-region geometry as the cause of Uranus' and Neptune's unusual magnetic fields. Nature 428(6979), 151-153 (2004). doi:10.1038/nature02376

56. Stanley, S., Bloxham, J.: Numerical dynamo models of Uranus' and Neptune's magnetic fields. Icarus 184(2), 556-572 (2006). doi:10.1016/j.icarus.2006.05.005

57. Stone, E.C., Cooper, J.F., Cummings, A.C., McDonald, F.B., Trainor, J.H., Lal, N., McGuide, R.E., Chenette, D.L.: Energetic particles in the uranian magnetosphere. Science 233, 93-97 (1986)

58. Stone, E.C., Miner, E.D.: The Voyager 2 encounter with the uranian system. Science 233, 3943 (1986). doi:10.1126/science.233.4759.39

59. Stone, E.C.: The Voyager 2 encounter with Uranus. J. Geophys. Res. 92(A13), 14873-14876 (1987). doi:10.1029/JA092iA13p14873

60. Tittemore, W.C., Wisdom, J.: Tidal evolution of the Uranian satellites-II. An explanation of the anomalously high orbital inclination of Miranda. Icarus 78, 63-89 (1989)

61. Tittemore, W.C., Wisdom, J.: Tidal evolution of the Uranian satellites. III-evolution through the Miranda-Umbriel 3:1, Miranda-Ariel 5:3, and Ariel-Umbriel 2:1 mean-motion commensurabilities. Icarus 85, 394-443 (1990). doi:10.1016/0019-1035(90)90125-S

62. Tittemore, W.C.: Tidal heating of Ariel. Icarus 87, 110-139 (1990). doi:10.1016/00191035(90)90024-4

63. Tóth, G., Kovács, D., Hansen, K.C., Gombosi, T.I.: Three-dimensional MHD simulations of the magnetosphere of Uranus. J. Geophys. Res. 109, A11210 (2004). doi:10.1029/ 2004JA010406

64. Trafton, L.M., Miller, S., Geballe, T.R., Tennyson, J., Ballester, G.E.: $\mathrm{H}_{2}$ Quadrupole and $\mathrm{H}_{3}^{+}$emission from Uranus: the Uranian thermosphere, ionosphere, and aurora. Astrophys. J. 524(2), 1059-1083 (1999). doi:10.1086/307838

65. Tsiganis, K., Gomes, R., Morbidelli, A., Levison, H.F.: Origin of the orbital architecture of the giant planets of the solar system. Nature 435, 459-461 (2005). doi:10.1038/nature03539

66. Vasavada, A.R., Showman, A.P.: Jovian atmospheric dynamics: an update after Galileo and Cassini. Rep. Prog. Phys. 68, 1935-1996 (2005). doi:10.1088/0034-4885/68/8/R06

67. Vasyliūnas, V.M.: The convection-dominated magnetosphere of Uranus. Geophys. Res. Lett. 13, 621-623 (1986). doi:10.1029/GL013i007p00621

68. Zarka, P., Lecacheux, A.: Beaming of Uranian nightside kilometric radio emission and inferred source location. J. Geophys. Res. 92, 15177-15187 (1987). doi:10.1029/ JA092iA13p15177

69. Zarka, P.: Auroral radio emissions at the outer planets: observations and theories. J. Geophys. Res. 103, 20159-20194 (1998)

70. Zarka, P.: Plasma interactions of exoplanets with their parent star and associated radio emissions. Planet. Space Sci. 55(5), 598-617 (2007). doi:10.1016/j.pss.2006.05.045 